\title{
Topological doping and the stability of stripe phases
}

\section{Citation}

Pryadko, Leonid P., Steven A. Kivelson, V. J. Emery, Yaroslaw B. Bazaliy, and Eugene A. Demler. 1999. "Topological Doping and the Stability of Stripe Phases." Physical Review B 60 (10) (September 1): 7541-7557. doi:10.1103/physrevb.60.7541.

\section{Published Version}

10.1103/PhysRevB.60.7541

\section{Permanent link}

http://nrs.harvard.edu/urn-3:HUL.InstRepos:28237644

\section{Terms of Use}

This article was downloaded from Harvard University's DASH repository, and is made available under the terms and conditions applicable to Other Posted Material, as set forth at http:// nrs.harvard.edu/urn-3:HUL.InstRepos:dash.current.terms-of-use\#LAA

\section{Share Your Story}

The Harvard community has made this article openly available.

Please share how this access benefits you. Submit a story.

Accessibility 


\title{
Topological doping and the stability of stripe phases
}

\author{
Leonid P. Pryadko \\ Institute for Advanced Study, Princeton, New Jersey 08540 \\ Steven A. Kivelson \\ Department of Physics \& Astronomy, University of California, Los Angeles, California 90095 \\ V. J. Emery \\ Department of Physics, Brookhaven National Laboratory, Upton, New York 11973-5000 \\ Yaroslaw B. Bazaliy \\ Department of Physics, Stanford University, Stanford, Calfornia 94305 \\ Eugene A. Demler \\ Institute for Theoretical Physics, University of California, Santa Barbara, Calfornia 93106-4030 \\ (Received 12 May 1999)
}

\begin{abstract}
We analyze the properties of a general Ginzburg-Landau free energy with competing order parameters, long-range interactions, and global constraints (e.g., a fixed value of a total "charge") to address the physics of stripe phases in underdoped high- $T_{c}$ and related materials. For a local free energy limited to quadratic terms of the gradient expansion, only uniform or phase-separated configurations are thermodynamically stable. "Stripe" or other nonuniform phases can be stabilized by long-range forces, but can only have nontopological (in-phase) domain walls where the components of the antiferromagnetic order parameter never change sign, and the periods of charge and spin-density waves coincide. The antiphase domain walls observed experimentally require physics on an intermediate length scale, and they are absent from a model that involves only long-distance physics. Dense stripe phases can be stable even in the absence of long-range forces, but domain walls always attract at large distances; i.e., there is a ubiquitous tendency to phase separation at small doping. The implications for the phase diagram of underdoped cuprates are discussed. [S0163-1829(99)03734-0]
\end{abstract}

\section{INTRODUCTION}

One of the fundamental issues in the theory of highly correlated solids is the nature of the ground-state phases produced when a small concentration $x$ of "doped holes" is introduced into a Mott insulator, particularly an antiferromagnet. It is now established ${ }^{1-4}$ that, at small enough $x$ and in the absence of long-range Coulomb interactions, a doped antiferromagnet generally phase separates into a hole-rich and a hole-free phase; i.e., the antiferromagnetic state is destroyed via a first-order phase transition. In the presence of weak, long-range Coulomb interactions that frustrate this local tendency to phase separation, the two-phase region is replaced by states which are inhomogeneous on intermediate length scales, ${ }^{4-6}$ and especially "stripe phases," which have now been observed in a wide variety of oxide materials. ${ }^{7-11}$ In various quasi-two-dimensional cuprate high-temperature superconductors and the isostructural nickelates the stripes are observed ${ }^{12}$ to be "topological," in the sense that the charge is concentrated along one-dimensional "rivers" which are at the same time antiphase domain walls in the antiferromagnetic $(\mathrm{AF})$ order. In the nearly cubic manganate colossal magnetoresistance materials, ${ }^{8,9}$ the "stripes" are two-dimensional sheets of charge which are nontopological. (In some sense, each sheet can be thought of as a dimer of topological stripes. ${ }^{9,13}$ )

Here we study the properties of a general Ginzburg-
Landau free energy with competing order parameters, longrange interactions, and global constraints [e.g., a fixed value of a total "charge," as defined in Eq. (2)] to address the physics of inhomogeneous ("stripe") phases. Specifically, a stripe phase is a unidirectional density wave which, in the case of a doped antiferromagnet, consists of a coupled spindensity wave (SDW) and charge-density wave (CDW). At very dilute doping, a stripe phase consists of an ordered array of far-separated self-localized structures or individual stripes. At moderate doping levels, where the spacing between stripes is comparable to their width, the structures are best described as nearly harmonic density waves.

Zachar and two of us ${ }^{14}$ have considered the density-wave limit of a Landau theory of coupled CDW and SDW order, each with a fixed wave vector $\vec{q}$, near a transition to a disordered state, which occurs as the temperature or doping is varied. The existence of a cubic term in the Landau free energy coupling these two order parameters drives the period of the SDW to be twice that of the CDW, and the absence of any net $\mathrm{AF}$ ordering is equivalent to the statement that the stripes are topological. By contrast, as shown in Appendix A, the same sort of term in the Landau theory of the transition between a homogeneous ordered antiferromagnetic phase and a stripe ordered phase produces a state in which the Néel magnetization does not change its sign between the domains; i.e., the stripes are nontopological.

To elucidate the circumstances in which arrays of stripes 
can be thermodynamically stable, and what determines their character (i.e., topological vs nontopological, collinear vs spiral) we shall concentrate on the dilute limit, where the spacing between stripes is large, and the stripes are highly anharmonic structures. Specifically, we study the extremal states of a general Ginzburg-Landau free energy functional for coupled order parameters as a function of the average charge density.

Whenever the order parameter profiles are slowly varying everywhere, so that only the lowest-order (quadratic) terms in the gradient expansion of the free energy are necessary [Eqs. (1) and (4)], we show the following.

(1) In the absence of long-range interactions, only spatially uniform and phase-separated (two-phase coexistence) states are globally stable.

(2) "Stripe" or other nonuniform phases can be stabilized by long-range forces, but they are nontopological in the sense that any component $u_{i}$ of the order parameter has a uniform sign as long as the free-energy density is an even function of $u_{i}$. [We indicate all point symmetry groups which satisfy this condition for a magnetic (pseudovector) order parameter.]

(3) Whenever there is a global rotational symmetry of the order parameter, any localized configuration which interpolates between two distinct asymptotic ground states (e.g., an antiphase domain wall) is locally unstable to untwisting.

The possibilities become richer in cases in which higherorder derivative terms in the Ginzburg-Landau free energy or lattice effects determine an additional length scale - the core size of a localized defect. ${ }^{14}$ When there is no frustration, topological stripes are still forbidden in the ground state. However, frustration, such as competing first- and secondneighbor interactions in a lattice model or opposite-sign terms in the gradient expansion of the Ginzburg-Landau model (i.e., below a Lifshitz point), can stabilize topological collinear domain walls. In the context of doped antiferromagnets, this kind of frustration can arise as a result of the competition between the tendency of the Coulomb interaction to localize the charges and the tendency of electrons to quantum delocalize. However, even in this case, the asymptotic interaction between defects is still attractive at large distances, so long-range forces are necessary to suppress phase separation in the dilute limit.

In other words, topological stripes are a consequence of physics on an intermediate length scale, and they do not appear in a theory that considers only long-distance or lowenergy physics.

The plan of this paper is as follows. In Sec. II we review some of the theoretical and experimental background. Specifically, we discuss some of the early theoretical work predicting stripe phases, the theoretical controversies concerning the range of phase separation in microscopic models, such as the $t-J$ model, and some of the experimental facts concerning stripe phases in doped antiferromagnetic insulators.

In Sec. III we perform a scaling analysis of possible nonuniform configurations which minimize a generalized Ginzburg-Landau functional, establish the analog of the virial theorem which relates the long-distance Coulomb interaction to the gradient energies of the system, and derive the universal asymptotic form of the large-distance interac- tions between domain walls or other defects.

In Sec. IV we analyze the local and global stability of nonuniform ground-state configurations. For systems with a global rotational symmetry of the order parameter, we show that the antiphase domain walls are locally unstable to "untwisting," even in the presence of long-range forces. If the rotational symmetry is broken, these domain walls can be locally stable, but they are not necessarily allowed in any ground-state configuration. We establish a corresponding sufficiency criterion for global instability for such antiphase domain walls, and identify the corresponding point symmetry groups of the underlying lattice.

In Sec. V, we show that antiphase domain walls can be stable even in the ground state, if the free-energy functional includes higher-derivative terms or is defined on the lattice. We discuss a sufficiency criterion for local stability of the solutions, and illustrate the effect of stabilization of antiphase domain walls in particular examples. We also show that, for systems with short-range interactions and mixed AF and charged order parameters, the domain walls always attract at large distances, which indicates a tendency to phase separation at small doping. If long-range Coulomb interactions are included as well, inhomogeneous phases are stabilized. Depending on the details, either wide stripes are produced via Coulomb-frustrated phase separation ${ }^{15,5}$ or certain dense stripe phases are stabilized, in agreement with the arguments of Hellberg and Manousakis. ${ }^{16,4}$

We conclude that although (avoided) phase separation is ubiquitous, especially at small doping, antiphase domain walls are not universal in the ground state, even in the presence of long-range forces. Certain types of short-distance physics are required to stabilize antiphase domain walls. Therefore, effective long-distance models are not, in general, sufficient for a successful description of the stripe morphology in the cuprates and nickelates.

\section{BACKGROUND}

The undoped parent compounds of the high- $T_{c}$ materials have one electron per unit lattice cell, and, if it were not for the electron-electron interactions, one would expect them to be metallic. Instead, strong Coulomb repulsion renders the system a Mott insulator and results in an AF ground state with a doubled unit cell. Unlike usual band insulators, such correlated insulators do not conduct even when weakly doped. The short-distance physics of the doped system, dominated by strong electron-electron repulsion, is believed to be captured in the large- $U$ Hubbard model, the $t-J$ model, ${ }^{17}$ or related models. ${ }^{18}$

Unfortunately, to this time, none of these models has been solved in anything resembling a physical regime of parameters. One well-established aspect is the tendency of these models to phase separation ${ }^{19-21,1-4}$ in a substantial range of parameters. In the presence of the long-range Coulomb repulsion phase separation is, of course, impossible, unless the dopants are mobile. Instead, the system forms a chargeinhomogeneous state, in which hole-rich regions exist in an antiferromagnetic background. ${ }^{4,5}$ Within this picture, it is natural to interpret the stripe phases observed in various doped antiferromagnets as being a consequence of Coulombfrustrated electronic phase separation (sometimes called mi- 
crophase separation ${ }^{9}$ ). Such stripe phases can be either metallic or insulating, depending on the character of the holerich phase. ${ }^{4,22-26}$ However, the precise range of parameters in which phase separation occurs in systems with short-range interactions and even the physical reasons for the stability of antiphase domain walls in systems with Heisenberg symmetry have not been fully elucidated. Moreover, phase separation, especially at small doping, is notoriously hard to see numerically; even for the most studied $t-J$ model, some numerical studies have been interpreted as indicative of ${ }^{20,2,3,27}$ the universality of phase separation in the limit of small doping, while others purport to indicate the existence of a critical ratio of $J / t$ below which phase separation does not occur. $^{28,24,25}$

For the case of doped AFs with unbroken spin-rotational invariance this controversy was resolved by Pryadko, Kivelson, and Hone. ${ }^{1}$ It was shown that spin-wave exchange always causes an attraction between localized holes or hole clusters, similar to the well-known Casimir effect. ${ }^{29}$ At large distances this attraction falls off as a power law, and therefore it is always stronger then the exponentially decreasing forces present in the system with short-range interactions. This proves that any phase with static charge order is thermodynamically unstable at small enough doping. However, the absolute magnitude of this attractive force is very small, and even a relatively weak easy-axis anisotropy (allowed by the symmetry in planar materials) can provide a spin-wave gap sufficient to suppress this effect.

Static incommensurate magnetic and charge order in the cuprate high-temperature superconductors was first discovered ${ }^{30}$ in $\mathrm{La}_{1.6-x} \mathrm{Nd}_{0.4} \mathrm{Sr}_{x} \mathrm{Cu} \mathrm{O}_{4+\delta}$. Recently, $x$-ray ${ }^{31-33}$ diffraction measurements have confirmed the existence of charge order. Moreover, in this material, static stripe order coexists ${ }^{34,35}$ with superconductivity, albeit with suppressed $T_{c}$. Additional indirect information about the frequency range of magnetic correlations was provided by local probes, such as muon spin resonance $(\mu \mathrm{SR}){ }^{36-38}$ In this material a structural phase transition to a low-temperature tetragonal (LTT) phase substantially stabilizes the stripe order, making it particularly easy to detect, but, at the same time, suppresses the superconducting transition temperatures. Indeed, in closely related materials (e.g., $\mathrm{La}_{1.4-x} \mathrm{Nd}_{0.6} \mathrm{Sr}_{x} \mathrm{Cu} \mathrm{O}_{4}$ ), static stripe order is observed, but no evidence of superconductivity has been found. ${ }^{39,40}$ However, more recently, static stripe order has been detected ${ }^{11}$ in the more widely studied high-temperature superconductors $\mathrm{La}_{2-x} \mathrm{Sr}_{x} \mathrm{Cu} \mathrm{O}_{4}$ with $0.05<x<0.13$ and ${ }^{10}$ "stage-IV" $\mathrm{La}_{2} \mathrm{CuO}_{4+\delta}$, in which the transition temperature $T_{c}$ $=42 \mathrm{~K}$ is not suppressed.

Moreover, evidence has mounted that in a still broader class of high-temperature superconductors (perhaps even all high-temperature superconductors) stripe order is nearly condensed in the sense that there are substantial stripelike correlations which persist at low temperatures over long intervals of space and time. Slow dynamically fluctuating incommensurate magnetic correlations were observed some time ago ${ }^{41}$ by inelastic neutron scattering in $\mathrm{La}_{2-x} \mathrm{Sr}_{x} \mathrm{Cu} \mathrm{O}_{4}$. That these incommensurate structures are simply fluctuating stripes is now clear from a comparison ${ }^{30,7}$ of the fluctuations in this material and its ordered cousin $\mathrm{La}_{1.6-x} \mathrm{Nd}_{0.4} \mathrm{Sr}_{x} \mathrm{Cu} \mathrm{O}_{4}$. Evidence supporting the universality of incommensurate fluctuations in high- $T_{c}$ materials has also been recently provided by neutron scattering studies ${ }^{42}$ of spin fluctuations in $\mathrm{YBa}_{2} \mathrm{Cu}_{3} \mathrm{O}_{7-x}$ and $\mathrm{Bi}_{2} \mathrm{Sr}_{2} \mathrm{CaCu}_{2} \mathrm{O}_{8}$, and indirect evidence of the same structures in $\mathrm{Bi}_{2} \mathrm{Sr}_{2} \mathrm{CaCu}_{2} \mathrm{O}_{8}$ has been obtained from angle-resolved photoemission spectroscopy (ARPES) ${ }^{43}$ Indirect evidence that static stripe structures may also be more common than previously appreciated can be deduced from $\mu \mathrm{SR}$ measurements ${ }^{44}$ and nuclear quadrupole resonance $(\mathrm{NQR})$ measurements. ${ }^{45}$

The existence of stripe phases was first established in the nickelates $\left(\mathrm{La}_{2-x} \mathrm{Sr}_{x} \mathrm{NiO}_{4+\delta}\right)$ by direct electron ${ }^{46}$ and neutron ${ }^{47-49}$ scattering. But the ubiquity of stripe phases in doped antiferromagnets has become clear only in the last couple of years of intensive experimental inquiry. Stripe order in the insulating, nearly cubic manganates has been vividly visualized by electron diffraction studies. ${ }^{9}$ Here the charge order is strongly coupled to a lattice (Jahn-Teller) distortion, which makes the stripes more classical and more strongly ordered; the stripes here are nontopological in the sense that the CDW period is equal to the SDW period. The real-space images constructed from the electron diffraction results make it clear that each nontopological stripe can be viewed as a pair of close-by topological stripes or, equivalently, that the topological stripe array has been dimerized.

In all cases in the cuprates and nickelates, where the information is available, the measured positions of the incommensurate peaks indicate that the period of spin modulation is twice that of the charge modulation. This and other data ${ }^{7}$ support the model ${ }^{50,51}$ of charged holes concentrated on the antiphase walls between neighboring antiferromagnetic domains. The effect of stabilization of such antiphase domain walls, or stripes, by the addition of charged holes to a correlated insulator, was named ${ }^{22}$ topological doping.

But while the existence of stripe phases in doped antiferromagnets is clearly established, and there is growing evidence that it is a general phenomenon, there is less agreement on the origins of the stripes and their implications. The existence of stripe phases consisting of arrays of antiphase domain walls in doped antiferromagnets was, in fact, predicted still earlier than the work ${ }^{15}$ on Coulomb frustrated phase separation on the basis of Hartree-Fock mean-field theory. ${ }^{50,52}$ The Hartree-Fock stripes always have a commensurate density of holes corresponding to one hole per site along the length of the stripes, and are always insulating; a gap equal to a substantial fraction of the insulating gap opens at the transition to the Hartree-Fock stripe phase. These are generalizations of similar calculations in one dimension ${ }^{53}$ to the higher-dimensional case, and are closely related to calculations ${ }^{54}$ which sought to explain the existence of strong incommensurate peaks in the magnetic susceptibility in terms of Fermi surface nesting; the stripe phase in HartreeFock theory is directly a consequence of that nesting. ${ }^{52}$ In detail, these approaches do not account for the behavior of the cuprates, in which the density of holes along a stripe varies ${ }^{55}$ continuously as a function of $x$, and the stripe phases are conducting or superconducting, not insulating. Moreover, the evidence from ARPES is that there are no sharply defined quasiparticles in the normal state of the cuprates. ${ }^{56}$ In the La-Sr-Cu-O family of materials, in which the evidence of stripe order and stripe fluctuations is strongest, there is simply no vestige of a quasiparticle in the region of momentum 
space where the nested Fermi surface is supposed to occur. ${ }^{43}$ However, these mean-field (MF) Hartree-Fock calculations already reflected the tendency ${ }^{57}$ of the holes to be collectively self-trapped in regions of suppressed antiferromagnetism, a close relative of phase separation. Moreover, they correctly identify the microscopic physics, the transverse kinetic energy of the holes, which gives rise to the antiphase character of the stripes.

The unreliability of the Hartree-Fock approximation for determining the properties of domain walls in strongly coupled systems was also pointed out by Nayak and Wilczek. ${ }^{23}$ They analyzed the energy per electron on a partially filled stripe, which, ignoring the effect of antiferromagnetic surrounding, was approximated as the sum of the energy of broken AF bonds and the kinetic energy of onedimensional electrons in the limit $U \rightarrow \infty$. Even in the absence of long-range interactions, the model does not develop a gap, and the value of the optimal filling of the stripes was shown to vary continuously with parameters. Therefore, the stripes in this approximation are conducting and not insulating as follows from the Hartree-Fock analysis.

An alternative phenomenology of high- $T_{c}$ materials was suggested by Zhang, ${ }^{58}$ who emphasized the competition between the superconducting and AF order parameters. In the vicinity of a (hypothetical) $\mathrm{SO}(5)$-symmetric point, where these two order parameters form a five-dimensional vector of "'superspin,' the effective free energy can be written in general Ginzburg-Landau form, with relatively small symmetry breaking terms. An analysis ${ }^{59}$ of nonuniform MF solutions in such a model (assuming that the magnitude of the fivedimensional "superspin" remains constant) was recently performed by Veillette et al. In the absence of the long-range Coulomb interaction, and at small enough doping, a Maxwell construction was used to show that the system phase separates into antiferromagnetic and superconducting regions. Turning on the long-distance Coulomb interaction stabilizes a variety of nonuniform droplet and stripe phases. Surprisingly (at the time), the expected antiphase domain walls were not discovered among the numerical solutions. The signs of both AF and SC order parameters were always uniform, although their magnitude changed substantially. It is apparent that the absence of antiphase domain walls is an artifact of the model, but the specific reason for this feature was not elucidated.

\section{MIXED PHASE OR PHASE SEPARATION}

\section{A. General scaling arguments}

The mean-field approach typically works well if the important degrees of freedom vary slowly in time and space. In such cases one can write an effective free energy in generalized Ginzburg-Landau form

$$
\mathcal{F}_{l}=\int d^{D} x\left\{\sum_{i}\left[\chi_{i}(\mathbf{u})\left(\nabla u_{i}\right)^{2}\right]+V(\mathbf{u})\right\},
$$

which retains only the leading (quadratic) terms in the expansion over the gradients of the order parameters $u_{i}$. Usually, such a form of the free energy [with $\chi=$ const and polynomial $V(\mathbf{u})]$ is used in the vicinity of a second-order phase transition, where the selection of the important terms is dic- tated by their "relevance" in the sense of an appropriate renormalization group flow. Similarly, in high-energy applications, ${ }^{60-62}$ only renormalizable potentials are usually considered. Here, we shall try to make as general an analysis as possible, and only assume that the positive susceptibilities $\chi_{i}(\mathbf{u})$ and the potential energy $V(\mathbf{u})$, which is bounded from below, are smooth enough functions of their arguments, so that a lowest-energy configuration always exists. Such a generalization of the Ginzburg-Landau free-energy functional is necessary because, as we shall show, form (1) is not sufficient for describing the stripe phases of interest, independently of the specific form of the local potential $V$.

The first statement is that the ground state of the model (1), possibly with one or more constraints of the form

$$
\mathcal{Q}=\int d^{D} x \rho(\mathbf{u})
$$

is either uniform or phase separated in the thermodynamic limit; the energy of any mixed (nonuniform) phase can always be lowered in an infinite system. To prove this, let us imagine that it were not the case and that some nonuniform configuration $\mathbf{u}=\mathbf{u}^{(1)}(x)$ (which, generally, we can assume to be periodic) minimizes the free-energy density $f=\mathcal{F} / \Omega$, and also, if necessary, satisfies the constraint for the charge density $\bar{\rho}=\mathcal{Q} / \Omega$. Then the dilated fields, $\mathbf{u}^{(\lambda)} \equiv \mathbf{u}^{(1)}(\lambda x)$ satisfy the same constraints, while the corresponding energy density

$$
f_{\lambda}=\lambda^{2} K^{(1)}+\Pi^{(1)},
$$

written here in terms of the original "kinetic" and "potential', energy densities

$$
K^{(1)} \equiv \int \sum_{i} \chi_{i}\left(\nabla u_{i}\right)^{2} \frac{d^{D} x}{\Omega}, \quad \Pi^{(1)} \equiv \int V(\mathbf{u}) \frac{d^{D} x}{\Omega},
$$

evaluated at the configuration $\mathbf{u}=\mathbf{u}^{(1)}(x)$, can be reduced by decreasing the scale parameter $\lambda$, which is equivalent to a uniform dilation of the original field configuration. This contradicts the original assumption, and we conclude that no such coordinate-dependent configuration can minimize the energy of the system.

It is important to emphasize that the statement proved above is only correct in the thermodynamic limit. For a periodic solution in a finite system the scaling parameter $\lambda$ can take only discrete values, so that at least one period would fit the system size. Further energy-density reduction is possible by doubling both the system size and the total charge, and then performing an additional rescaling. Such scaling also has a direct implication for possible numerical studies of this and related models: because $\lambda^{2} K^{(1)} \sim 1 / L^{2}$, the finite-size correction to the free energy and other parameters will be likely to fall off as a power of the system size.

At first sight it appears that the existence of stable kinks for any symmetric double-well potential contradicts this statement. We must point out, however, that only a singlekink solution is topologically stable; in any configuration with periodic boundary conditions one has an equal number of kinks and antikinks, and the energy can be lowered by annihilating the pairs. For periodic potentials, multikink configurations may be topologically stable, as long as the total 
number of kinks is fixed by the boundary conditions. With free or periodic boundary conditions, however, such extremal solutions never represent the ground state of the system.

Similarly, one can create stable nontopological solitons ${ }^{62-67}$ by minimizing the energy of the system with an imposed finite-charge, as opposed to a finite-charge-density, constraint. In this case the amount of charge itself is used to introduce an additional length scale which fixes the size of the soliton, and the question about phase separation does not arise. The solution of this apparent paradox is that, if the thermodynamic limit is defined correctly, both the energy (1) and the conserved charge (2) will turn out to be infinite (or zero), and they cannot be used to define a length scale. Only in this case the correct procedure is to minimize the finite density of the system's free energy, at a given charge density.

Let us now consider how the scaling in Eq. (3) is modified in the presence of a long-range interaction

$$
\mathcal{F}_{C}=\int d^{D} x d^{D} x^{\prime} \frac{[\rho(\mathbf{u}(x))-\bar{\rho}]\left[\rho\left(\mathbf{u}\left(x^{\prime}\right)\right)-\bar{\rho}\right]}{\left|x-x^{\prime}\right|^{\gamma}},
$$

where $\gamma<D$ for convergence. Obviously, in this case the total charge constraint (2) can be dropped, because the integration in Eq. (4) will diverge in large systems if the screening is not perfect, no matter how weak the interaction is. Evaluating the free-energy density along the dilated field configuration $u^{(\lambda)}$ (which, of course, must have the correct value of the average charge density, so that the long-range part of the energy is finite) we obtain, instead of Eq. (3),

$$
f_{\lambda}=\lambda^{2} K^{(1)}+\Pi^{(1)}+\lambda^{-D+\gamma} V^{(1)},
$$

where $V^{(1)}$ is the long-range energy (4) per unit volume, evaluated for the field configuration $\mathbf{u}^{(1)}$. The integral (4) converges if $D-\gamma>0$, and the free-energy density $f_{\lambda}$ has a minimum at $\lambda=1$ if

$$
2 K=(D-\gamma) V .
$$

This expression is analogous to the virial theorem ${ }^{68}$ for the considered class of models. It is the manifestation of the equilibrium between competing gradient terms, which tend to dilate the system, and the long-range forces, which tend to decrease the scale of charge variations. As a result of this competition, an additional length scale is introduced into the problem, and periodic field configurations can be stabilized. ${ }^{69}$

\section{B. Interaction of defects}

Despite its generality, the scaling technique, considered above, is limited to continuous models. Furthermore, it is not sensitive enough for analyzing the stability of more general models, where the existence of mixed phases may depend on actual parameters. Indeed, if the shape of individual solitonlike or instantonlike defects for a given model is fixed at some short scale, the mixed phase can often be understood as a lattice of such relatively weakly coupled defects. The stability of such a phase will be defined by soundlike displacement modes, which are likely to be much softer than the uniform dilations we considered so far. The relevant elasticity modulus will obviously be defined by the interaction between the constituent defects.

In this section we discuss how the asymptotic form of the interaction between widely separated solitons can be found by a simple linear analysis, even though the core structure of the solitons themselves is governed by a complicated set of nonlinear differential equations. Qualitatively, this is so because away from their cores solitons asymptotically approach one of the uniform "vacuum" configurations, and the interaction between two solitons, placed sufficiently far apart, can depend only on the form of this asymptotic falloff. Indeed, the mutual interaction can be interpreted as a force exerted on the core of either soliton in the presence of the infinitesimal field created by the other; therefore, this interaction cannot depend on the internal structure of either soliton as long as the large-distance asymptotic form remains the same.

This implies that the interaction between individual solitons must be totally determined by the region of overlapping tails. In this region the amplitude of the perturbation of the vacuum is small, and the effective free energy can be linearized. After this step, the linearized problem reduces to a static Schrödinger equation in an external potential, and the interaction energy can be found by standard methods. ${ }^{70}$

As an illustration, ${ }^{71}$ consider a one-dimensional $(D=1)$ free energy of the form (1), with constant susceptibilities $\chi_{i}=1 / 2$, and the potential $V(\mathbf{u}) \geqslant 0$ reaching global minima only at $\mathbf{u}_{ \pm}= \pm \mathbf{m}, V( \pm \mathbf{m})=0$. In the absence of any special symmetries, there exists only one (up to translations) minimal-energy trajectory $\mathbf{u}^{0}(x)$ interpolating between these minima, $\mathbf{u}^{0}( \pm \infty)= \pm \mathbf{m}$. With this trajectory, we can also construct approximate double-kink trajectories of the form

$$
\mathbf{u}(x)=\mathbf{u}^{0}\left(x-x_{1}\right)+\mathbf{u}^{0}\left(x_{2}-x\right)-\mathbf{m},
$$

and write the corresponding interaction energy as

$$
\begin{aligned}
\delta \mathcal{F} & \equiv \mathcal{F}\left[\mathbf{u}_{1}+\mathbf{u}_{2}-\mathbf{m}\right]-\mathcal{F}\left[\mathbf{u}_{1}\right]-\mathcal{F}\left[\mathbf{u}_{2}\right] \\
& =\int_{-\infty}^{\infty} d x\left[\mathbf{u}_{1}^{\prime} \mathbf{u}_{2}^{\prime}+V\left(\mathbf{u}_{1}+\mathbf{u}_{2}-\mathbf{m}\right)-V\left(\mathbf{u}_{1}\right)-V\left(\mathbf{u}_{2}\right)\right],
\end{aligned}
$$

where $\mathbf{u}_{1,2}=\mathbf{u}^{0}\left( \pm x \mp x_{1,2}\right)$, and the prime denotes the spatial derivative. Let us choose a point $x_{0}$ somewhere between the positions of the kinks, $x_{1} \ll x_{0} \ll x_{2}$. Then, in the left domain $x<x_{0}$ the field $\delta \mathbf{u}_{1} \equiv \mathbf{u}_{2}-\mathbf{m}$ is small and can be considered as a small perturbation, while in the region $x>x_{0}$ the field $\delta \mathbf{u}_{2} \equiv \mathbf{u}_{1}-\mathbf{m}$ is small. Keeping only the terms of linear order in each domain, we obtain

$$
\begin{aligned}
\delta \mathcal{F}= & \int_{-\infty}^{x_{0}} d x\left\{\left(\mathbf{u}_{1}^{\prime} \delta \mathbf{u}_{1}\right)^{\prime}+\delta \mathbf{u}_{1}\left[-\mathbf{u}_{1}^{\prime \prime}+\frac{\partial}{\partial \mathbf{u}} V\left(\mathbf{u}_{1}\right)\right]\right\} \\
& +\int_{x_{0}}^{\infty} d x\{1 \leftrightarrow 2\},
\end{aligned}
$$

or just

$$
\delta \mathcal{F}=\mathbf{u}_{1}^{\prime}\left(\mathbf{u}_{2}-\mathbf{m}\right)-\left.\mathbf{u}_{2}^{\prime}\left(\mathbf{u}_{1}-\mathbf{m}\right)\right|_{x=x_{0}},
$$

where the bulk terms disappear to this order because each field $\mathbf{u}_{1}$ and $\mathbf{u}_{2}$ obeys the Euler-Lagrange extremum equa- 
tions exactly. Despite appearances, the interaction energy (9) is actually independent of the choice of the point $x_{0}$, as long as it is located far enough from the cores of the kinks, so that the linearized Euler-Lagrange equations apply.

Equation (9) relates the long-distance interaction between the kink and the antikink with their asymptotic form at large distances. For multicomponent order parameters the asymptotic properties may vary. However, in the particular case of antisymmetric kinks, $\mathbf{u}_{0}(x)=-\mathbf{u}_{0}(-x)$, we can choose the separation point $x_{0}=\left(x_{2}+x_{1}\right) / 2$ exactly midway between the kinks, and the interaction energy can be rewritten as

$$
\begin{aligned}
\delta \mathcal{F} & =\left.2 \mathbf{u}_{0}^{\prime}\left(\mathbf{u}_{0}-\mathbf{m}\right)\right|_{x=L / 2} \\
& =\left.\frac{d}{d x}\left(\mathbf{u}_{0}-\mathbf{m}\right)^{2}\right|_{x=L / 2}<0,
\end{aligned}
$$

where $L=x_{2}-x_{1}$ is the distance between the kinks, and the negative sign of the derivative corresponds to a positive quantity asymptotically vanishing far to the right of the kink. The obtained sign corresponds to an attraction at large distances. The attraction is also expected for a pair of symmetric nontopological solitons (in this case the same formula with an appropriate $\mathbf{m}$ applies). Of course, for the case of a single-component order parameter, $\mathbf{u} \equiv u$, this result is well known. Even in a more general case, we could expect to find the attraction between such defects, as we already know that inhomogeneous configurations are always thermodynamically unstable in the system (1), (2), unless there are topological reasons for the stability. The effect of topological stability is also easy to understand here: equally charged kinks [which are allowed, for example, if the potential $V(u)$ is periodic] always repel. In accordance with Sec. III A, such kinks would be pushed infinitely far apart unless stabilized by the boundary conditions.

A similar calculation can be repeated for any combination of spatially separated defects, in arbitrary dimension. In every case the interaction in the lowest order can be split into a sum of pairwise terms which are defined by the gradient terms in the original free energy.

\section{SYMMETRY AND THE STRUCTURE OF DOMAIN WALLS}

So far we mostly considered global properties of the configurations minimizing the free energy of the general form (1). For this local functional we saw that nonuniform states are unstable to phase separation, and thus indicated the Coulomb repulsion as an important component of any continuous mean-field model designed to describe the observed incommensurate structures in high- $T_{c}$ materials. Now let us concentrate on the local structure of nonuniform configurations minimizing the free energy (1), (4). Specifically, we shall attempt to answer the question whether a component of the order parameter can change its sign in a thermodynamically stable state (ground-state configuration).

For this question to make sense, the zero value must have an unambiguous meaning. This is guaranteed if the free energy depends only on the square of the order parameter. For example, in antiferromagnets time-reversal symmetry assures that this is the case for the pseudovector of magnetiza- tion s. Even if the full spin-rotational symmetry is broken, the susceptibilities $\chi_{i}$, the potential $V$, and the charge density $\rho$ can only depend on the bilinear combinations $s_{i} s_{j}$ of the magnetization components. The free energy will depend only on the squares $s_{i}^{2}$ as long as the mixed combinations with $i$ $\neq j$ are prohibited by the symmetry, as discussed in Sec. IV C.

\section{A. Continuous symmetry and the untwisting instability}

Let us first consider a system with a free energy of the form (1), (4), with an additional rotational symmetry between $m \geqslant 2$ components of the order parameter $\mathbf{u}$ $=\left(s_{1}, \ldots, s_{m}, \phi_{1}, \ldots\right)$. For clarity, and having in mind a particular application to magnets, we shall call these the components of a (generalized) spin magnetization $\mathbf{s}$, and assume that both local and nonlocal parts of the free energy can only depend analytically on the square $S^{2} \equiv \mathbf{s}^{2}$ of this vector, while the dependence on the remaining components $\phi_{i}$ remains generic,

$$
\rho(\mathbf{u}) \equiv \rho\left(\mathbf{s}^{2}, \phi_{1}, \ldots\right), \quad V(\mathbf{u}) \equiv V\left(\mathbf{s}^{2}, \phi_{1}, \ldots\right), \ldots
$$

In the presence of such continuous spin-rotational symmetry, the gradient terms in the free energy (1) tend to align the direction of the magnetization $\mathbf{s}$. Indeed, the rotationally symmetric gradient term can be written as

$$
\chi_{s}\left(S^{2}, \phi_{i}\right)(\nabla \mathbf{s})^{2}=\chi_{s}\left(S^{2}, \phi_{i}\right)\left[(\nabla S)^{2}+S^{2}(\nabla \hat{\mathbf{e}})^{2}\right]
$$

where $\hat{\mathbf{e}} \equiv \mathbf{s} / S$ is a unit vector in the direction of $\mathbf{s}$. Obviously, in any region where $S \neq 0$, the energy of a "twisted" configuration ( $\hat{\mathbf{e}} \neq$ const $)$ can be lowered by aligning the magnetization along a common direction, which eliminates the second term on the right-hand side (RHS) of Eq. (10). The rotational stiffness vanishes if $S=0$ (nodal points in onedimensional case or nodal hypersurfaces for $D>1$ ), and the energy does not depend on the relative orientation of the vectors $\mathbf{S}$ in the regions separated by such nodes. In any case, one can select $s_{1}= \pm S, s_{l}=0$ for $l>1$; i.e., the minimal configuration can be always chosen to have only one component, although the sign of this component is not fixed at this point. We shall show below, however, that the energy of any such configuration with a node (closed nodal surface for $D>1$ ) can be continuously lowered by introducing an appropriately chosen perturbation in the orthogonal direction. Such instability to local "untwisting", is well known for onedimensional systems; it implies that only uniformly oriented spin configurations can minimize the free energy in the presence of a rotational symmetry.

To analyze the "untwisting", instability in general, consider a spin configuration $\mathbf{s}=\left(s_{0}, 0\right)$ with a single nonzero component $s_{0}(x)$ which is presumed to have a node (nodal surface for $D>1$ ). The local instability of such configurations can be demonstrated by introducing an orthogonal perturbation $\mathbf{s}_{1}=\left(0, s_{1}\right)$. The relevant part of the perturbed free energy functional (1) can be written as

$$
\mathcal{F}=\int d^{D} x\left\{\chi\left(S^{2}, x\right)\left[\left(\nabla s_{0}\right)^{2}+\left(\nabla s_{1}\right)^{2}\right]+V\left(S^{2}, x\right)\right\}
$$


where $S^{2}=s_{0}^{2}+s_{1}^{2}$, and the additional coordinate dependence is introduced to account for a possible presence of the remaining nonuniform components of the order parameter. Here we only consider a simpler case in which the charge density $\rho$ (and, consequently, the long-range Coulomb interaction) is independent of the spin configuration; this is generalized in Appendix C.

To quadratic order in the perturbation $s_{1}$ the increment of the free energy (11) is

$$
\delta \mathcal{F}=\int\left\{\chi_{0}(x)\left(\nabla s_{1}\right)^{2}+G_{0}(x) s_{1}^{2}\right\} d^{D} x,
$$

where the effective susceptibility $\chi_{0}(x) \equiv \chi\left(s_{0}^{2}, x\right)>0$ is positive everywhere, the effective potential $G_{0}(x)$ $\equiv \chi^{\prime}\left(s_{0}^{2}, x\right)\left(\nabla s_{0}\right)^{2}+V^{\prime}\left(s_{0}^{2}, x\right)$ is continuous and limited from below, and primes denote derivatives with respect to $S^{2}$. The local stability of the configuration $s_{0}(x)$ requires that the functional $\delta \mathcal{F}$ be non-negative; equivalently, the selfadjoint eigenvalue problem

$$
-\nabla\left(\chi_{0}(x) \nabla \varphi\right)+G_{0}(x) \varphi=\Lambda \varphi
$$

should have no negative eigenvalues. Using the spinrotational symmetry (or directly, by comparing with the Euler-Lagrange equation for $s_{0}$ ), it is easy to see that the function $\varphi_{0}(x) \equiv$ const $\times s_{0}(x)$ satisfies Eq. (12) with zero eigenvalue $\Lambda_{0}=0$. It is a well-known fact about the selfconjugate eigenvalue problem (12) that its ground state is nondegenerate and does not change sign. ${ }^{72}$ Since the function $\varphi_{0}(x)$ does change its sign by assumption, it cannot be the ground-state eigenfunction, and, therefore, there must be at least one unstable direction $\varphi_{-1}(x)$ which corresponds to a lower eigenvalue $\Lambda_{-1}<\Lambda_{0}=0$. Therefore, the energy of the original spin configuration $\left(s_{0}(x), 0\right)$ can be continuously lowered by the orthogonal perturbation $\mathbf{s}_{1}=$ const $\times\left(0, \varphi_{-1}(x)\right)$, and we conclude that only a uniformly oriented spin configuration without nodes (nodal hypersurfaces for $D>1$ ) can realize the global minimum of the functional (1) in the presence of a continuous spin-rotation symmetry. ${ }^{73}$

\section{B. Instability in the Ising limit}

Let us now imagine that the continuous spin-rotational symmetry is broken by the lattice. We begin with the case of a relatively strong easy-axis (Ising) anisotropy, so that effectively only one component $s$ of the spin remains. In the absence of any other magnetic ordering, the residual symmetry of the free energy is the discrete $Z_{2}$ group associated with the time-reversal symmetry $s \rightarrow-s$. Ordinarily, such broken symmetry indicates the possibility of topologically stable kinks, or domain walls in $D>1$, separating regions of opposite magnetization. It turns out, however, that despite their local stability, such configurations do not occur in the lowest-energy state of the system; they can only occur as excitations. Formally, this can be proved in general, utilizing the residual symmetry $Z_{2}$ of the free-energy functional.

Indeed, we saw that in the presence of a continuous spinrotational symmetry the ground-state configuration is uniformly aligned; it can always be chosen to have only one component of the spin. Therefore, the ground state of the functional

$$
\mathcal{F}=\int d^{D} x\left\{\chi\left(s^{2}, x\right)(\nabla s)^{2}+V\left(s^{2}, x\right)\right\},
$$

is in a one-to-one correspondence (modulo the overall rotation) with the ground state of the U(1)-symmetric extended functional

$$
\mathcal{F}_{\mathbf{z}}=\int d^{D} x\left\{\chi\left(\mathbf{s}^{2}, x\right)(\nabla \mathbf{s})^{2}+V\left(\mathbf{s}^{2}, x\right)\right\},
$$

where the field $\mathbf{s}=\left(s_{1}, s_{2}\right)$ has two components. Because of the untwisting instability, the second functional has a nodeless ground-state configuration; our mapping indicates that so does the first.

We have proved a version of the no-node theorem, i.e., the statement that any component $s$ of the order parameter preserves its sign in the globally minimal configuration, provided that the potential energy (including the long-distance part; see Appendix C) depends only on the square of this component.

\section{Group-theoretical analysis: Effects of "spin-orbit coupling,'}

The situation of perfect Ising anisotropy considered in the previous section is, of course, an idealized case. In real systems the anisotropy can be quite small, so that all three components $\left(s_{x}, s_{y}, s_{z}\right)$ of the magnetization pseudovector must be considered. Nevertheless, it is possible to show that the same conclusion about the absence of topological domain walls holds as long as the symmetry of the underlying lattice is high enough.

Generally, because of the global time-reversal symmetry, the local potential energy can be an arbitrary function of all bilinear combinations $s_{i} s_{j}, i, j=x, y, z$. Expanding in powers of such products, we can also write any such function as

$$
V\left(s_{i} s_{j}\right)=V_{0}+V_{1} s_{y} s_{z}+V_{2} s_{z} s_{x}+V_{3} s_{x} s_{y},
$$

where the coefficients in the expansion are, generally, some functions of the squares of the magnetization components, $V_{k} \equiv V_{k}\left(s_{x}^{2}, s_{y}^{2}, s_{z}^{2}\right), k=0, \ldots, 3$. The statement about the sign of the magnetization components proved in the previous section applies only if the cross terms are absent. In particular, this happens independently of the specific details of the function $V\left(s_{i} s_{j}\right)$, if such terms are not allowed by the symmetry of the lattice. Conversely, if at least one of such terms is present, no general statement about the sign of any component of the spin magnetization can be made, unless the additional components of magnetization are suppressed by a sufficiently strong easy-axis anisotropy.

The effective free-energy functional should remain invariant under any transformation which preserves the lattice structure; for the local potential $V$ only the transformations from the corresponding crystallographic point group are relevant. Because the pseudovector of magnetization remains invariant under inversion, its components transform under reflection,

$$
\sigma_{h}: \quad(x, y, z) \rightarrow(x, y,-z),
$$

as $\left(s_{x}, s_{y}, s_{z}\right) \rightarrow\left(-s_{x},-s_{y}, s_{z}\right)$, in exactly the same fashion as under the $\pi$ rotation with respect to the axis $z$, 


$$
C_{2}: \quad(x, y, z) \rightarrow(-x,-y, z)
$$

The invariance of the potential (13) with respect to either of these transformations requires $V_{1}=V_{2}=0$. The existence of another symmetry transformation of one of these kinds, with respect to an orthogonal plane or an orthogonal axis, is sufficient to suppress the only remaining coefficient, $V_{3}=0$.

Such symmetries are present in all crystallographic point groups of cubic (groups $O, O_{h}, T, T_{h}, T_{d}$ ) and orthorhombic $\left(C_{2 v}, D_{2}, D_{2 h}\right)$ systems, and in sufficiently symmetric groups of tetragonal $\left(C_{4 v}, D_{4}, D_{4 h}, D_{2 d}\right)$ and hexagonal $\left(C_{6 v}, D_{6}, D_{6 h}, D_{3 h}\right)$ systems. For all other crystallographic groups we constructed invariant expressions, mixing several components of the magnetization. For example, the quantity $s_{x} s_{y}\left(s_{x}^{2}-s_{y}^{2}\right)$ is symmetric with respect to all transformations of the groups $C_{4}, C_{4 h}$, and $S_{4}$, the quantity $s_{z} s_{y}\left(s_{y}^{2}-3 s_{x}^{2}\right)$ is symmetric with respect to all trigonal groups, etc.

The lattice symmetry also determines the structure of the derivative terms in the free-energy functional. In addition to components of the pseudovector of the magnetization, $\mathbf{s}$, we now have the components of the axial vector of the gradients, and so the number of possible symmetric terms increases. The conclusions about the phase separation and the local structure of the domain walls will be absolutely modified if the terms linear in derivatives are present in the free energy. Such terms are known to stabilize topological domain walls in the ground state. Among the groups we listed above, only the groups $O_{h}, T_{h}, D_{6 h}, D_{4 h}$, and $D_{2 h}$ absolutely prohibit the existence of invariant quantities linear in derivatives. All these groups include the inversion, which guarantees the absence of such invariants. The groups which include only proper rotations were eliminated by the existence of the pseudoscalar invariant $\mathbf{s} \cdot[\nabla \times \mathbf{s}]$. All other groups required special consideration. ${ }^{75}$

The highly symmetric point groups listed in the previous paragraph prohibit both terms linear in derivatives, and the mixing between different components of the magnetization in the potential energy. Nevertheless, in the presence of a spin-orbit interaction any point symmetry group allows mixing between different components of the magnetization in the gradient terms due to the existence of a rotationally invariant scalar

$$
(\nabla \cdot \mathbf{s})^{2}=\left(\partial_{x} s_{x}\right)^{2}+2 \partial_{x} s_{x} \partial_{y} s_{y}+\cdots
$$

For specific groups, dangerous terms can also include lesssymmetric invariant quantities containing terms of the form $\partial_{x} s_{x} \partial_{y} s_{y}$. Formally, because these terms cannot be eliminated by symmetry, antiphase domain walls are possible in the ground state of any non-Heisenberg system. For the case of magnetic ordering one may argue, however, that the symmetry breaking in the gradient terms can only result from the combination of the hopping, already small because it is determined by the tunneling matrix elements, and the spin-orbit interaction, typically small because it is a relativistic effect. Therefore, such terms are expected to be very small, and it is clear that they cannot be responsible for very robust antiphase domain wall ordering observed in the cuprates and nickelates.

\section{ANTIPHASE DOMAIN WALLS}

The crystallographic point groups of the relevant phases of high- $T_{c}$ materials $^{76-80}$ and related compounds ${ }^{81,82}$ are $D_{4 h}$ in tetragonal phases and $D_{2 h}$ in orthorhombic phases. According to our arguments in the previous section, these highly symmetric groups absolutely rule out antiphase domain walls in the lowest-energy state, and yet such domain walls have been observed in many such materials. Moreover, this constraint is not limited to the continuous model (1) with gradient terms quadratic in derivatives: many lattice models with arbitrary long-distance interactions can be cast in the generic form considered in Appendix D, and by the theorem proved there they must have ground states with uniform sign of the order parameter. Clearly, this situation is by no means an exception.

For example, a tendency for forming in-phase domain walls was seen ${ }^{83}$ in a model of two Heisenberg antiferromagnets coupled across a stripe represented by a Luttinger liquid, as a result of their interaction with the staggered magnetic moments induced on the stripe. This is not surprising in view of our general MF treatment; the model ${ }^{83}$ ignores completely the transverse mobility of the stripe (the processes of electrons' hopping from AF to the stripe) which counteracts the usual exchange coupling. ${ }^{84,85}$

In the remaining part of the paper we show that antiphase domain walls in the ground state can be stabilized in the presence of frustration involving competing interactions. We consider two specific models with short-range interactions: a lattice model of a doped antiferromagnet and a continuous model with higher-order derivative terms. In both systems periodic antiphase domain wall structures can be thermodynamically stable at large enough charge densities, but domain walls attract at asymptotically large distances, so that the phase separation necessarily happens at sufficiently small values of doping.

\section{A. Antiphase domain walls on the lattice}

Let us consider a lattice model of the form

$$
\mathcal{F}=J \sum_{\langle i j\rangle} \mathbf{S}_{i} \mathbf{S}_{j}+J^{\prime} \sum_{\langle i l j\rangle} \mathbf{S}_{i} n_{l} \mathbf{S}_{j}+\sum_{i} V\left(\mathbf{S}_{i}^{2}, n_{i}\right),
$$

where the first term represents the usual exchange of localized spins, the second term ${ }^{84,85}$ is due to higher-order exchange processes with virtual hops through a partially occupied site, the hole density, $0 \leqslant n_{l} \leqslant 1$ is defined to be a bounded continuous variable, and the local potential $V$ must be chosen to ensure the stability of the model, as well as to provide an adequate repulsion between the holes and the spins on the same site. As usual, we presume that the average hole density is fixed,

$$
x \equiv \bar{n}=\mathcal{N}^{-1} \sum_{i} n_{i},
$$

where $\mathcal{N}$ is the total number of lattice sites. Clearly, the positive values of the second exchange constant, $J^{\prime}>0$, tend to frustrate antiferromagnetic ordering in a doped system; we argue below that a competition of this sort is necessary to form antiphase domain walls and suppress the global AF order in the system. 


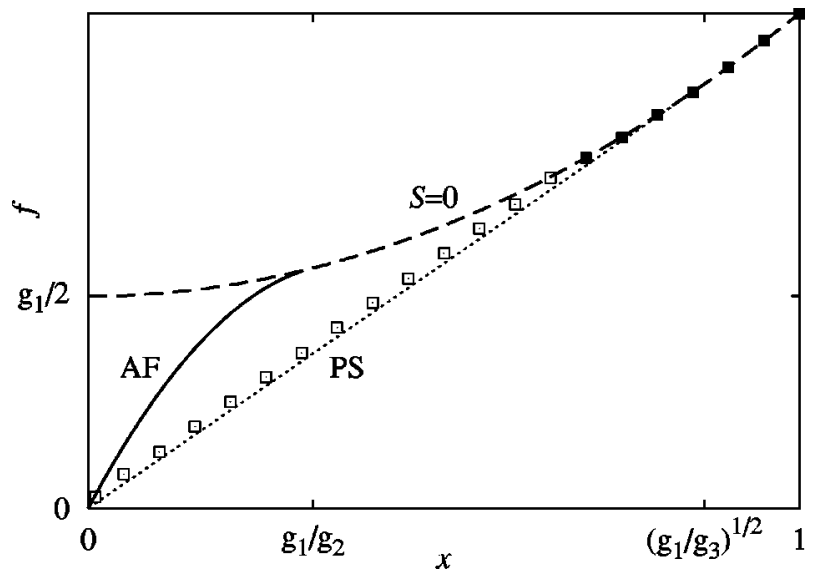

FIG. 1. Locating the minimum of the free energy (17) per unit site for the strong repulsion case, $J=0.9, J^{\prime}=0.6, g_{1}=0.6, g_{2}$ $=1.9$, and $g_{3}=0.8$. Bold solid and dashed lines respectively show the energies of uniform AF and nonmagnetic $(S=0)$ phases. The dotted line gives the free energy per site of an infinite system in the phase-separated regime. Solid and open squares respectively indicate periodic and phase-separated configurations minimized numerically with system sizes up to $\mathcal{N}=40$.

For the purpose of this example, we will limit our analysis to the quartic form of the potential

$$
V\left(\mathbf{S}^{2}, n\right)=\frac{g_{1}}{2}\left(\mathbf{S}^{2}-1\right)^{2}+\left(\widetilde{g}_{2} n+\frac{z J}{2}\right) \mathbf{S}^{2}+\frac{g_{3}}{2} n^{2},
$$

where $\tilde{g}_{2}=g_{2}-z(z-1) J^{\prime} / 2, z$ is the lattice coordination number, and the coefficients are chosen so that in terms of the antiferromagnetic Néel order parameter $\mathbf{s}_{i}=(-1)^{i} \mathbf{S}_{i}$ the free energy could be rewritten in a form

$$
\begin{aligned}
\mathcal{F}= & \frac{J}{2} \sum_{\langle i j\rangle}\left(\mathbf{s}_{i}-\mathbf{s}_{j}\right)^{2}+J^{\prime} \sum_{\langle i l j\rangle} n_{l}\left(\mathbf{s}_{i} \mathbf{s}_{j}-\mathbf{s}_{l}^{2}\right) \\
& +\sum_{i}\left[\frac{g_{1}}{2}\left(\mathbf{s}_{i}^{2}-1\right)^{2}+g_{2} n_{i} \mathbf{s}_{i}^{2}+\frac{g_{3}}{2} n_{i}^{2}\right] .
\end{aligned}
$$

The term with the coefficient $g_{1}$ favors unit values of the on-site magnetization, and the coefficient $g_{2}$ is a measure of the strength of the repulsion between spins and charges, while the coefficient $g_{3}$ measures the local tendency against doping.

At zero doping all charges necessarily vanish, $n_{l}=0$, and Eq. (17) is minimized by a uniform AF state $\mathbf{s}^{2}=1$ with the value $\mathcal{F}_{\mathrm{AF}}(0)=0$. Uniform $\mathrm{AF}$ states can be also formally found at sufficiently small nonzero dopings, with energy given by the second line of Eq. (17), minimized at $\mathbf{s}^{2}=1$ $-g_{2} x / g_{1} \geqslant 0$ with the energy-density value

$$
f_{\mathrm{AF}}(x)=g_{2} x+\frac{x^{2}}{2}\left(g_{3}-\frac{g_{2}^{2}}{g_{1}}\right) \text {. }
$$

The magnitude of the AF ordering reduces to zero at $x$ $=g_{1} / g_{2}$, and at larger filling fractions the AF phase is replaced by a uniform nonmagnetic state with the energy $f_{0}$ $=\left(g_{1}+g_{3} x^{2}\right) / 2$.

The energies of these phases for the strong repulsion case $g_{2}^{2}>g_{1} g_{3}$ are illustrated in Fig. 1. The function $f_{\mathrm{AF}}(x)$ (solid

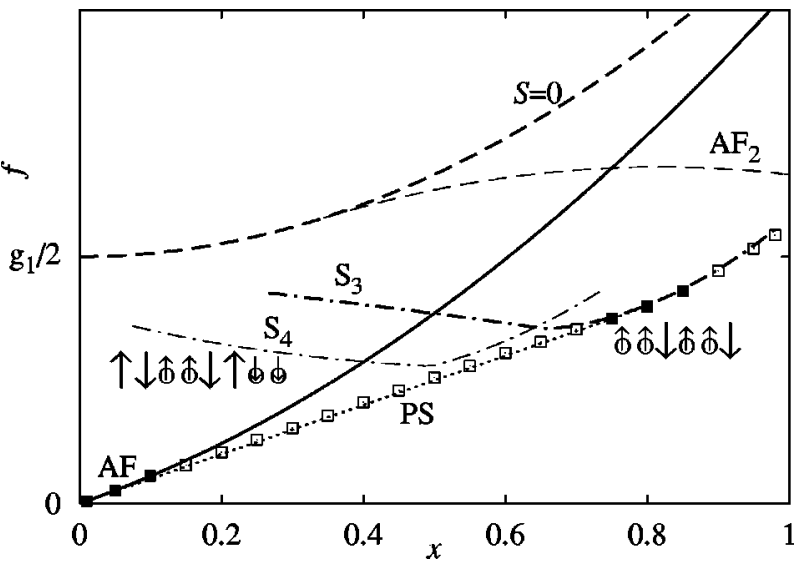

FIG. 2. Locating the minimum of the free energy (17) per unit site for the case of weak repulsion, $J=0.9, J^{\prime}=0.6, g_{1}=0.6, g_{2}$ $=0.3$, and $g_{3}=0.8$. The line $\mathrm{AF}_{2}$ corresponds to a uniform $\mathrm{AF}$ with the period of four lattice sites, which becomes preferable at larger values of $J^{\prime}$. The lines $S_{3}$ and $S_{4}$ correspond to commensurate stripe phases with the charge periods 3 and 4 , as illustrated in the insets. Below $x \approx 0.75$ the system phase separates into an undoped (or very weakly doped) AF phase and the phase $S_{3}$. Solid and open squares respectively indicate the phase-separated and uniform configurations as seen numerically with system sizes up to $\mathcal{N}=40$.

line) has a negative curvature at small values of doping, so the system is necessarily unstable to phase separation between an undoped antiferromagnet and a completely or partially doped uniform nonmagnetic phase (dashed line). The energy of phase-separated system is shown in Fig. 1 with a dotted line. The absence of other phases was checked numerically by minimizing Eq. (17) for systems with periodic boundary conditions of all even sizes in the range between $\mathcal{N}=4$ and $\mathcal{N}=40$. To reduce the possibility of accidental trapping in a local minimum, we used the Metropolis algorithm with variable temperature (simulated annealing). For each system size we did a set of up to eight trial cooldown runs starting with a random configuration, selected the best resulting configuration, and then repeatedly cycled the temperature up to 20 times. The minimal energy density chosen among the systems of all sizes was used as an estimate of the ground state energy; these values are shown in Figs. 1 and 2 with squares. As expected, in the regime of phase separation, typically the lowest energy density was achieved for the biggest system.

Phase separation is impossible if a long-distance interaction is also included in the model (14). However, the above calculation remains relevant as long as this interaction is sufficiently weak. In this case, there exists a large length scale $D$, at which the long-distance forces become relevant. It is this scale that determines the period of a stripe phase, in which the regions of undoped $\mathrm{AF}$ and nonmagnetic phases are separated by the domain walls of the model (14). As long as the size $d$ of these domain walls is relatively small, $d$ $\ll D$, the long-range interaction does not significantly change their form.

In the considered regime of the strong local repulsion, $g_{2}^{2} \gg g_{1} g_{3}$, the domain wall between the undoped AF and non-magnetic phases with density $x=\min \left[1,\left(g_{1} / g_{3}\right)^{1 / 2}\right]$ is very sharp. The order parameters approach their vacuum values as determined by the solution of the corresponding lin- 
earized equations. On the AF side, the charge density is locked at $n=0$, and the perturbation $\delta \mathbf{s}_{j} \sim \exp \left(-\kappa_{0} j\right)$ falls off with the same exponent as in the ideal undoped AF,

$$
\sinh ^{2}\left(\kappa_{0} / 2\right)=g_{1} / J
$$

Similarly, expanding the free energy (17) to quadratic order in the vicinity of the zero-magnetization state with the density $n_{1}=\left(g_{1} / g_{3}\right)^{1 / 2}<1$, we obtain

$$
\begin{aligned}
4 \sinh ^{2}\left(\kappa_{1} / 2\right)= & \left(2-\frac{J}{2 n_{1} J^{\prime}}\right) \\
& +\sqrt{\left(2-\frac{J}{2 n_{1} J^{\prime}}\right)^{2}+\frac{2\left(g_{2} n_{1}-g_{1}\right)}{n_{1} J^{\prime}}} .
\end{aligned}
$$

The second term under the square root, and, consequently, the RHS of the entire expression, are guaranteed to remain positive everywhere in the strong repulsion regime, independent of the values of the exchange constants. The domain walls are relatively narrow when $\kappa D \ll 1$; in this case the solution has a form of an array of domain walls between the AF and nonmagnetic regions. This is the canonical picture of Coulomb-frustrated phase separation, ${ }^{15,5}$ where wide stripes are directly analogous to the classical stripe phases. ${ }^{6}$

The ground-state phase diagram changes substantially in the opposite case of very weak repulsion, $g_{2}^{2} \ll g_{1} g_{3}$. The main difference of this regime is that nonuniform phases with antiphase domain walls are much closer to stability; as illustrated in Fig. 2, some of them may be stable even in the absence of any long-range forces. As the long-range interactions are introduced, instead of stabilizing wide stripes by the usual Coulomb-frustrated phase separation ${ }^{15,5}$ mechanism, they may stabilize certain dense stripe phases. Such a picture of Coulomb-stabilized microscopic stripe phases is in agreement with the arguments of Hellberg and Manousakis ${ }^{16}$ based on their results of exact numerical diagonalization of small $t-J$ clusters.

In the considered limit of weak repulsion, $g_{2}^{2} \ll g_{1} g_{3}$, nonzero magnetization can coexist with substantial doping even in the limit of a fully doped system, $x=1$. Because of the constraint $0 \leqslant n_{i} \leqslant 1$, only a uniform charge configuration is possible at $x=1$, and the spin ordering is determined by the competition between two exchange couplings. For a particular set of parameters chosen in Fig. 2, the lowest-energy phase in this limit has a spin modulation period of three lattice sites. As the doping is reduced, it is energetically favorable to put all electrons at the points of maximum magnetization, so that the charge density has a period of three lattice sites, as illustrated in the right caption. The energy of such a ferrimagnetic phase $S_{3}$ is denoted with a bold dashdotted line in Fig. 2; as the doping is lowered, this line starts to increase again below the point $x \approx 0.67$ where single undoped sites are separated by fully doped antiphase domain walls of width two sites. In a similar phase $S_{4}$ (with the charge period of four and the spin period of eight sites), such domain walls are separated by two weakly doped sites, but this phase is avoided in large systems which prefer to phase separate instead. The energy density of a phase-separated system (PS) is shown with the dotted line; in the vicinity of the point $x \sim 0.5$ this line goes only slightly below the line denoting the energy of the stripe phase, $S_{4}$.

Numerically, for all combinations of parameters we tried, the nonuniform "stripe" phases seemed to be stable only at sufficiently large values of doping. It turns out that this statement can be proved for any form of the potential $V\left(\mathbf{s}^{2}, n\right)$ in Eq. (14) by using a variant of the argument in Sec. III B. Any nonuniform charge configuration in the limit of low doping must consist of some defects, charged solitons or domain walls, separated by wide regions of almost perfect AF. In this limit every defect, described by the spin $\mathbf{s}_{i}$ and charge $n_{i}$ distributions, must realize a local minimum of the free energy (14), and satisfy appropriate Euler-Lagrange equations. A two-defect configuration can be well approximated by a linear superposition of corresponding spin- and charge-density distributions, with the value of the constraint (15) independent of the mutual position of the defects. In the vicinity of each defect the effect of the other one can be considered as a perturbation. By rearranging the sums independently in each region, with the help of the corresponding Euler-Lagrange equations, the linear order cross terms can be made to disappear in the bulk, so that only the "integrated" part

$$
\begin{aligned}
\delta E= & +J \delta s_{0}^{b} \delta s_{1}^{a}+J^{\prime}\left[s_{-1}^{a} n_{0}^{a} \delta s_{1}^{b}-\delta s_{0}^{b} n_{1}^{a} s_{2}^{a}\right]-J \delta s_{0}^{a} \delta s_{1}^{b} \\
& -J^{\prime}\left[s_{-1}^{b} n_{0}^{b} \delta s_{1}^{a}-\delta s_{0}^{a} n_{1}^{b} s_{2}^{b}\right]
\end{aligned}
$$

remains. Here $\delta s \equiv s-s_{\infty}$ is the deviation of the AF magnetization from its vacuum value, and the superscripts $a$ and $b$ label the fields caused by the defect situated far to the left and far to the right from the origin, respectively. Similarly to Eq. (9), the precise location of the separation boundary is not important, as long as it is chosen far enough from each defect. For a symmetric defect configuration $s_{l}^{a}=s_{1-l}^{b}$, Eq. (19) can be rewritten as

$$
\begin{aligned}
\delta E= & J\left[\left(\delta s_{1}^{a}\right)^{2}-\left(\delta s_{0}^{a}\right)^{2}\right]+2 J^{\prime} s_{\infty}^{a}\left[\delta n_{0}^{a} \delta s_{0}^{a}-\delta n_{1}^{a} \delta s_{1}^{a}\right] \\
& +2 J^{\prime} n_{\infty}^{a}\left[\delta s_{-1}^{a} \delta s_{0}^{a}-\delta s_{1}^{a} \delta s_{2}^{a}\right],
\end{aligned}
$$

where $\delta n_{l} \equiv n_{l}-n_{\infty}$. Only the first term exists for the asymptotic form (18), where the hole density $n_{l}$ is pinned to zero at finite distances from defects. This term gives a negative interaction energy, corresponding to asymptotic attraction between far-separated defects. This is in accordance with our simulation in Fig. 2, where the most stable chargemodulated configuration was a dense condensate of antiphase stripes. Of course, the repulsion of the stripes at small distances and the stability of the dense stripe configuration cannot be inferred from this asymptotic analysis.

Generally, for models of the form (14), the hole density $n_{l}$ does not necessarily vanish at a finite distance from a defect, or it may even have a nonzero value $n_{\infty}$ in the intermediate AF phase. Then the second exchange term also contributes to the interaction energy. In principle, this contribution may be attractive or repulsive, depending on the relative sign of $s_{\infty} \delta s$ and $\delta n$. However, we are interested in systems with a strong repulsion between AF ordering and the doped holes; here the effect of the second exchange is negative, and the second term in the first line of Eq. (20) gives attraction as well. 
Contrarily, the last term in Eq. (20), which exists only if the doping saturates to a nonzero value $n_{\infty}$ far from the solitons, is positive; it contributes to a repulsion between the domain walls. This is not surprising, because the second exchange term counteracts the usual exchange if a finite hole density is present. Nevertheless, one can show that the net result is an attraction between the defects, as long as the uniformly doped AF state is locally stable.

\section{B. Antiphase domain walls in a continuum model}

Although we now have an example of a model which admits antiphase domain walls in the ground state, this model is not a continuum model, and one might infer that it is the lattice commensuration effects that enable the existence of antiphase domain walls in the ground state. To stress our statement that it is not the lattice, but the frustration between different interactions that stabilizes such domain walls, we give a brief analysis of a continuum model with similar properties.

Consider a one-dimensional system with the free energy of the form

$$
\mathcal{F}=\int d x\left[\beta\left(\mathbf{s}^{\prime \prime}\right)^{2}+\chi_{s}\left(\mathbf{s}^{\prime}\right)^{2}+\chi_{\phi}\left(\phi^{\prime}\right)^{2}+V\left(\mathbf{s}^{2}, \phi\right)\right] .
$$

As usual, the primes denote spatial derivatives, the field $\mathbf{s}$ represents an antiferromagnetic order parameter, and $\phi$ is a scalar field with some conserved charge density $\rho=\rho(\phi)$. Unlike Eq. (1), we no longer assume that the spin susceptibility $\chi_{s}=\chi_{s}(\phi)$ is a positively defined function of the scalar order parameter $\phi$, and the higher-order derivative term, with $\beta>0$, is required for stability. In analogy with the second hopping term of the lattice model (14), we shall assume that the spin susceptibility

$$
\chi_{s}(\phi)=1-\alpha \rho(\phi)
$$

depends linearly on the charge density, so that its sign can be reversed in the presence of large enough hole density.

\section{Scaling analysis}

It is obvious that the general conclusion of instability of periodic states made in Sec. III A does not apply for the model (21). Indeed, instead of Eq. (3), we obtain

$$
f_{\lambda}=\lambda^{4} Q_{1}+\lambda^{2} K_{1}+\Pi_{1},
$$

where $Q_{1}>0$ is the contribution of the term(s) quartic in the derivatives. Because the second-derivative terms are no longer positively defined, this expression may have a minimum at $\lambda=1$ and

$$
K_{1}=-2 Q_{1}<0 .
$$

Although this condition does not guarantee the global stability of a periodic solution, it is clear that periodic structures may in principle be stabilized for the free energy (23).

\section{Asymptotic interaction of domain walls}

The asymptotic form of the interaction between the domain walls for the model (21) can be easily found by a linear analysis similar to that in Sec. III B, by evaluating the energy of a superposition of two domain walls separated by a wide stretch of undoped antiferromagnet. As before, only surface terms survive in the linear order,

$$
\begin{aligned}
\delta E= & 2 \beta \delta \mathbf{s}_{a}^{\prime \prime} \delta \mathbf{s}_{b}^{\prime}-2\left(\beta \delta \mathbf{s}_{a}^{\prime \prime}\right)^{\prime} \delta \mathbf{s}_{b}+2 \chi_{s} \delta \mathbf{s}_{a}^{\prime} \delta \mathbf{s}_{b}+2 \chi_{\phi} \phi_{a}^{\prime} \phi_{b} \\
& -(a \leftrightarrow b),
\end{aligned}
$$

where the scalar field $\phi_{i}$ and the deviation of the AF order parameter $\delta \mathbf{s}_{i}$ must satisfy the corresponding Euler-Lagrange equations exactly; $i=a, b$ respectively denotes the defect located far to the left and far to the right of the point where this expression is evaluated. For two symmetric domain walls $\mathbf{s}_{a}(x)=\mathbf{s}_{b}\left(2 x_{0}-x\right)$ this expression is simplified if the point $x_{0}$ is chosen exactly in the middle,

$$
\delta E=-4\left(\beta \delta \mathbf{s}_{a}^{\prime \prime} \delta \mathbf{s}_{a}\right)^{\prime}+2 \chi_{s}\left(\delta \mathbf{s}_{a}^{2}\right)^{\prime}+\left.2 \chi_{\phi}\left(\phi_{a}^{2}\right)^{\prime}\right|_{x=x_{0}} .
$$

The parameters $\beta, \chi_{s}$, and $\chi_{\phi}$ in this expression must be evaluated in the vacuum configuration; they are all positive. The perturbation of the vacuum state gets smaller as we move to the right, and the two last terms are negative; as before, this corresponds to an attractive interaction. However, it is easy to see that the first term is positive; it contributes to the repulsion between the domain walls. Only by analyzing the linearized Euler-Lagrange equations in the nearly perfect $\mathrm{AF}$ region can we conclude that the overall sign of the interaction energy is negative, as long as the $\mathrm{AF}$ state is a locally stable minimum of the functional (21). Therefore, as previously, domain walls attract at large enough distances, and the system cannot form a stable nonuniform solution at asymptotically small doping as long as $\mathrm{AF}$ ground state is stable at zero doping and as long as there are no long-range forces.

\section{Twist stability}

The twist instability, which was discussed in Sec. IV for positive $\chi_{s}$ and $\beta=0$, can be also avoided for the model (21); a magnetization vector $\mathbf{s}$ can reverse its direction and yet remain locally stable with respect to twists. A sufficient condition for this stability can be obtained by analyzing the derivative terms in the free energy (21). By decomposing the vector $\mathbf{s}=S \mathbf{e}$ into a product of its magnitude $S$ and the unit vector $\mathbf{e}$, after several integrations by parts, the gradient terms in the free energy can be rendered into a form

$$
\begin{gathered}
\left(\mathbf{s}^{\prime \prime}\right)^{2} \rightarrow S^{2}\left(\mathbf{e}^{\prime \prime}\right)^{2}+\left(\mathbf{e}^{\prime}\right)^{2}\left[2\left(S^{\prime}\right)^{2}-4 S^{\prime \prime} S\right]+\left(S^{\prime \prime}\right)^{2}, \\
\left(\mathbf{s}^{\prime}\right)^{2}=\left(S^{\prime}\right)^{2}+S^{2}\left(\mathbf{e}^{\prime}\right)^{2} .
\end{gathered}
$$

The system (21) will remain stable to developing spontaneous twists as long as the coefficient in front of $\left(\mathbf{e}^{\prime}\right)^{2}$ remains positive; this gives the sufficiency criterion of stability, namely, the condition that the expression

$$
2 \beta\left(S^{\prime}\right)^{2}-4 \beta S^{\prime \prime} S+\chi_{s} S^{2}>0
$$

must remain positive everywhere. This condition is easy to check directly for any given single-component solution of Euler-Lagrange equations; there is no need to look for multicomponent solutions if Eq. (24) is satisfied.

Formally, this expression can remain positive near a node of the magnetization because of the presence of the higher- 
derivative term in Eq. (21). However, such solutions can be allowed in the ground state only if $\chi_{s}$ can become negative, which indicates the presence of a competition between different interactions. Therefore, the role of the higherderivative term is only to limit the instability caused by this competition.

\section{Approximate variational solution}

To illustrate the considered general properties, let us choose the potential

$$
V=\int\left[\frac{g_{1}}{2}\left(\mathbf{s}^{2}-1\right)^{2}+g_{2} \rho \mathbf{s}^{2}+\frac{g_{3}}{2} \rho^{2}\right] d x,
$$

of the same quartic form as used in Eq. (17), with $\rho$ $\equiv \rho(\phi)=\phi^{2}$. Numerically, the solutions at small enough densities look very much like the usual domain walls in magnets, with $\mathbf{s}$ changing its sign where $\phi$ has a maximum. Although the simplest set of trial functions $\phi=\phi_{0} / \cosh (k x)$, $s=\tanh (k x)$ does not work, we can use it as a variational solution to estimate the ground-state energy and the areas of stability of different phases.

Performing the integration, we obtain the expression for the total charge,

$$
\mathcal{Q}_{0}=\int d x \phi^{2}=2 \phi_{0}^{2} / k,
$$

and the free energy,

$$
\begin{aligned}
\mathcal{F}_{0}= & \frac{2}{3 k}\left[g_{1}+\frac{8}{5} \beta k^{4}+k^{2}\left(2+\phi_{0}^{2}-\frac{8}{5} \alpha \phi_{0}^{2}\right)\right. \\
& \left.+\phi_{0}^{2}\left(g_{2}+g_{3} \phi_{0}^{2}\right)\right] .
\end{aligned}
$$

In the limit of small charge density the stripe solution must minimize the energy per unit doped charge, $f_{0} \equiv \mathcal{F}_{0} / \mathcal{Q}_{0}$. This is achieved by selecting the amplitude of the charge soliton

$$
\phi_{0}^{4}=\left(g_{1}+2 k^{2}+8 \beta k^{4} / 5\right) / g_{3} .
$$

The resulting expression has a minimum at a nonzero scale $k=k_{0}$ if the constant $\alpha$ in Eq. (22) is

$$
\alpha=\frac{5}{8}\left[1+\frac{2 \sqrt{g_{3}}\left(1+8 \beta k_{0}^{2} / 5\right)}{\sqrt{g_{1}+2 k_{0}^{2}+8 \beta k_{0}^{4} / 5}}\right] ;
$$

the corresponding value of the energy per unit charge is

$$
f_{0}=g_{2}+\frac{2 \sqrt{g_{3}}\left(g_{1}+k_{0}^{2}\right)}{\sqrt{g_{1}+2 k_{0}^{2}+8 \beta k_{0}^{4} / 5}} .
$$

The resulting configuration will be stable with respect to twists if the criterion (24) is satisfied. The analysis shows that this is indeed the case for large enough values of $\beta$ and $g_{3}$.

The stability of a stripe phase made out of these domain walls is determined by Eq. (23). With the derived expressions we find that

$$
K_{0}=\left(1-\frac{8}{5} \alpha\right)+\frac{2}{\phi_{0}^{2}}=-\frac{16 \beta k^{2}}{5 \phi_{0}^{2}}
$$

is always negative. This implies that the periodic phase might indeed be stabilized at some intermediate scale, in agreement with our numerical simulations of this model. Therefore, the local stability of topological domain walls may lead to the stabilization of a dense stripe phase made out of such walls, in agreement with detailed simulations ${ }^{24,25}$ of the $t-J$ model. However, such a phase can only be stable at large enough charge densities: within the MF approximation we have shown that the asymptotic large-distance interaction between such domain walls is always attractive, and in the limit of small values of doping the system necessarily phase separates. In addition, more subtle fluctuation effects ${ }^{1}$ always contribute to power-law Casimir attraction between charged defects, and the statement about the phase separation in weakly doped antiferromagnets persists.

\section{CONCLUSIONS}

Phase separation at small doping is a ubiquitous property of doped insulators with short-distance interactions. Generally, in the absence of a frustration caused by competing interactions, the staggered magnetization of the ground state never changes its sign. These two statements can be formulated as theorems in the vicinity of a second-order phase transition involving $\mathrm{AF}$ ordering, where the correlation length is large and the derivative terms are small.

In application to high- $T_{c}$ materials, the competition between the tendency of the holes to move around and the tendency of repulsive interactions to localize the charges must be accounted for in any model for describing high- $T_{c}$ superconductors or related materials. Only at relatively short distances (where, strictly speaking, we go outside the limits of applicability of the MF theory) may the domain walls repel, which could lead to the stabilization of dense static stripe phases.

\section{ACKNOWLEDGMENTS}

We would like to thank M. Fogler and S.-C. Zhang for valuable discussions. L.P.P. was supported in part by the Grant No. DOE DE-FG02-90ER40542. S.A.K. was supported in part by the NSF under Grant No. DMR 98-08685 at UCLA. V.J.E. was supported by Department of Energy Grant No. DE-AC02-98CH10886. Y.B.B. was supported in part by Grant No. DMR 9814289 and under an IBM Research Partnership Award. E.A.D. was supported by NSF at ITP.

\section{APPENDIX A: LANDAU THEORY}

The phase transition between a stripe phase and a hightemperature disordered state considered by Zachar et al. ${ }^{14}$ involves only one spin-order parameter, the incommensurate spin-density wave $\mathbf{S}_{\mathbf{q}}$. The transition from a well-developed antiferromagnet with a modulation vector $\vec{\pi}=(\pi, \pi)$ to an incommensurate modulated phase must account for both the original AF-order parameter $\mathbf{S}_{\vec{\pi}}$ (which, generally, cannot be assumed small) and the spin-density wave $\mathbf{S}_{\vec{\pi}+\mathbf{k}}$, with modu- 
lation period $2 \pi / k$. Coupling these two spin-order parameters together, it is easy to write nontrivial and yet spinrotation-invariant terms of the Landau expansion of the effective free energy,

$$
\mathcal{F}=r_{\mathrm{s}}\left|\mathbf{S}_{\vec{\pi}+\mathbf{k}}\right|^{2}+r_{\mathrm{c}}\left|\rho_{\mathbf{k}}\right|^{2}+\gamma\left[\mathbf{S}_{\pi}^{*} \mathbf{S}_{\vec{\pi}+\mathbf{k}} \rho_{\mathbf{k}}^{*}+\text { c.c. }\right]+\cdots,
$$

where $\rho_{\mathbf{k}}$ is the complex-valued amplitude of the chargedensity wave with the wave vector $\mathbf{k}, \rho_{\mathbf{k}}^{*} \equiv \rho_{-\mathbf{k}}$, and the quartic (and higher-order) terms required for stability are omitted. This expression suggests that an instability in either the spin $\left[r_{\mathrm{s}} \equiv r_{\mathrm{s}}(\mathbf{q})<0\right]$ or the charge $\left[r_{\mathrm{c}} \equiv r_{\mathrm{c}}(\mathbf{k})<0\right]$ sector generates both spin- and charge-density waves at the wave vectors $\mathbf{q}=\vec{\pi}+\mathbf{k}$ and $\mathbf{k}$, respectively, with modulation amplitudes linearly proportional to each other. More precisely, the modulation appears if $r_{\mathrm{s}}(\mathbf{q})$ and/or $r_{\mathrm{c}}(\mathbf{k})$ are negative, or if

$$
r_{\mathrm{s}}(\vec{\pi}+\mathbf{k}) r_{\mathrm{c}}(\mathbf{k})<|\gamma|^{2}\left|\mathbf{S}_{\vec{\pi}}\right|^{2} .
$$

Near the transition the magnitude of the incommensurate peak is necessarily much smaller than the commensurate $\mathrm{AF}$ modulation, $\left|\mathbf{S}_{\vec{\pi}+\mathbf{k}}\right| \ll\left|\mathbf{S}_{\ddot{\pi}}\right|$; it is easy to see that this corresponds to in-phase domain walls. The derived relationship between $\mathbf{q}$ and $\mathbf{k}$ implies that the periods of spin and charge modulation must be equal for such domain walls.

Experimentally, novel incommensurate elastic peaks, coexisting with the commensurate peaks at $(\pi, \pi)$, have been observed $^{86}$ recently at the border of the antiferromagnetic region of $\mathrm{La}_{2-x} \mathrm{Sr}_{x} \mathrm{Cu} \mathrm{O}_{4}$ at $x=0.05$. The incommensurate peaks are rotated by $45^{\circ}$ compared to the antiphase peaks at larger doping, which could be caused by the fact that these peaks appear at a temperature that is lower than the energy of the low-temperature-orthorhombic-low-temperature-tetragonal phase mode. ${ }^{87}$ If the data represent a bulk effect, and assuming that the commensurate AF correlation length in the cluster spin-glass phase ${ }^{44}$ at smaller values of doping $(x$ $<5 \%$ ) is sufficiently large for the Landau expansion (A1) to apply, we interpret the simultaneous presence of both commensurate and incommensurate peaks as the signature of inphase domain walls, expected in this region, and not merely coexisting antiferromagnetic and stripe phases. The above analysis indicates that the corresponding charge modulation must have the same period and direction as that of the SDW order. Because the observed ordering differs substantially for these two phases, the transition from a weakly modulated diagonal AF phase to the fully developed stripe state with antiphase domain walls is expected to be first order in a clean system.

In general, however, a discontinuous transition between a topological and nontopological stripe phases is not the only possibility. A particularly simple scenario of a continuous transition between these phases corresponds to a dimerization transition, where pairs of antiphase domain walls spontaneously merge to form wider dimerized domain walls, similar to those observed in manganates. ${ }^{9}$ As a result, the period of charge modulation doubles, and a CDW with the periodicity of the original spin ordering must develop. In addition, the perfect symmetry between the regions with two opposite signs of AF order is broken, and a net antiferromagnetic ordering appears. Here we present only the simplest scenario for such a continuous transition, minimally extending the charge-driven part of the phase diagram of Zachar et al. ${ }^{14} \mathrm{~A}$ more complete symmetry analysis of possible dimerized phases will be published elsewhere. ${ }^{88}$

To describe the dimerization transition, the Landau effective free energy must include at least two harmonics of the density wave, $\rho_{\mathbf{k}}, \rho_{2 \mathbf{k}}$, coupled to the SDW harmonics $\mathbf{S}_{\pi+l \mathbf{k}}, l=0,1,2$. While the quadratic part of the free energy has the usual form,

$$
\mathcal{F}_{2}=\sum_{l=0}^{2} r_{s l}\left|\mathbf{S}_{\vec{\pi}+l \mathbf{k}}\right|^{2}+\sum_{l=1}^{2} r_{\rho l}\left|\rho_{l \mathbf{k}}\right|^{2}
$$

there are five possible cubic terms

$$
\begin{aligned}
\mathcal{F}_{3}= & \rho_{2 \mathbf{k}}^{*}\left(\lambda_{0} \mathbf{S}_{\vec{\pi}+\mathbf{k}}^{2}+\lambda_{1} \rho_{\mathbf{k}}^{2}+\lambda_{2} \mathbf{S}_{\pi} \mathbf{S}_{\vec{\pi}+2 \mathbf{k}}\right) \\
& +\rho_{\mathbf{k}}^{*}\left(\gamma_{0} \mathbf{S}_{\vec{\pi}+\mathbf{k}} \mathbf{S}_{\vec{\pi}}+\gamma_{1} \mathbf{S}_{\vec{\pi}+2 \mathbf{k}} \mathbf{S}_{\vec{\pi}+\mathbf{k}}^{*}\right)+\text { c.c. }
\end{aligned}
$$

The invariant with the coefficient $\lambda_{0}$ has been considered previously in Ref. 14, and the terms with coefficients $\gamma_{0}$ and $\lambda_{2}$ were considered above in Eq. (A1).

Let us follow Zachar et al. ${ }^{14}$ and consider the transition from a disordered phase, driven by the instability in the CDW sector, $r_{\rho 2}<0$. In this scenario, as the amplitude of the CDW $\rho_{2 \mathbf{k}}$ gets sufficiently large, the term with the coefficient $\lambda_{0}$ generates an instability in the SDW sector. From our extended free energy (A2) it is clear that the same density wave may also destabilize the double-periodic $\operatorname{CDW} \rho_{\mathbf{k}}$ (via the term with coefficient $\lambda_{1}$ ). If this is the case, the remaining cubic invariants will simultaneously generate nonzero AF modulation $\mathbf{S}_{\vec{\pi}}$ (coefficient $\gamma_{0}$ ) and an additional SDW harmonic $\mathbf{S}_{\vec{\pi}+2 \mathbf{k}}$ (coefficients $\lambda_{2}$ and $\gamma_{1}$ ). Obviously, in a certain range of parameters, the transition to the phase with $\rho_{\mathbf{k}} \neq 0$ is continuous. The resulting dimerized phase, with equal periods of SDW and CDW, and a nonzero AF ordering, would be interpreted as a nontopological stripe phase. If observed, such a transition will provide a precise macroscopic meaning to the notion ${ }^{9}$ of dimerized stripes.

\section{APPENDIX B: INTERACTION OF CHARGED SOLITONS}

Here we demonstrate that the expression for interaction energy between the defects, derived in Sec. III B, also works for systems with global charge constraint (2). The singlesoliton field configuration $\mathbf{u}_{0}(Q ; x)$ minimizes the energy functional at a fixed value of charge $Q$, but the total charge corresponding to their linear superposition (7) does not necessarily equal $2 Q$. Therefore, instead of Eq. (7), we need to consider a corrected configuration

$$
\begin{aligned}
\mathbf{u}(x) & =\mathbf{u}^{0}\left(Q-\delta Q ; x-x_{1}\right)+\mathbf{u}^{0}\left(Q-\delta Q ; x_{2}-x\right)-\mathbf{m} \\
& =\mathbf{u}^{0}\left(x-x_{1}\right)+\mathbf{u}^{0}\left(x_{2}-x\right)-\mathbf{m}+\delta \mathbf{u}(x),
\end{aligned}
$$

where the additional exponentially small (of the order of the tail overlap $\delta Q$ ) deformation

$$
\delta \mathbf{u}=-\delta Q\left[\frac{\partial \mathbf{u}^{0}\left(Q ; x-x_{1}\right)}{\partial Q}+\frac{\partial \mathbf{u}^{0}\left(Q ; x_{2}-x\right)}{\partial Q}\right]
$$

serves to adjust the value of charge constraint, so that, e.g., 


$$
\delta Q_{L}=\int_{-\infty}^{x_{0}}\left(\mathbf{u}_{2}-\mathbf{m}+\delta \mathbf{u}\right) \frac{\partial \rho\left(\mathbf{u}_{1}\right)}{\partial \mathbf{u}_{1}} d x=0,
$$

and a similar condition for the region $x>x_{0}$ where the field $\mathbf{u}_{2}$ is far from equilibrium value (all notations as in Sec. III B). In the presence of the charge constraint the EulerLagrange equations for a single kink must be written with a chemical potential $\mu$,

$$
-\mathbf{u}_{0}^{\prime \prime}+\left.\frac{\partial}{\partial \mathbf{u}}[V(\mathbf{u})+\mu \rho(\mathbf{u})]\right|_{\mathbf{u}=\mathbf{u}_{0}}=0
$$

and the combination in the square brackets in the integrand of Eq. (8) no longer disappears. Instead, it changes the energy by an amount proportional to the total charge increment $\delta Q_{L}$ in the region $x<x_{0}$ and a similar term for $x>x_{0}$. These charge increments vanish for the corrected configuration (B1), and in the linear order we are again left with the same universal expression (9). As before, it was important that the correct configuration deviate very little from the simple minded superposition (7), including the tail regions, where the correction $\delta \mathbf{u}$ can be safely ignored as an exponentially small quantity of higher order.

Such linear analysis is equivalent to finding the instantaneous acceleration ${ }^{89,90}$ of a defect surrounded by a surface by calculating the total flux of the energy-momentum tensor into the enclosed volume due to all other defects located outside the surface. The corrections to Eq. (9) are easy to find in equilibrium, and they indeed turn out to be exponentially smaller, if the locally stable configuration of several defects exists (in some cases such configurations can be stabilized by the boundary conditions). Often, however, because of the attraction between individual solitons, there are no locally stable equilibrium configurations minimizing the free energy. In such cases, instead of analyzing the forces in static configurations, the interaction can be found more accurately by studying the full dynamics of the system. ${ }^{91}$ In the present work, however, we are mostly interested in the sign of the interaction between defects, and the accuracy of Eq. (9) is sufficient.

\section{APPENDIX C: UNTWISTING INSTABILITY OF CHARGED DEFECTS}

Here we extend the local stability analysis of Sec. IV A to systems with conserved charge and long-range interactions. Now, instead of Eq. (11), the relevant part of the free energy and the corresponding constraint can be written as

$$
\begin{gathered}
\mathcal{F}=\int \chi\left(S^{2}, x\right)(\nabla \mathbf{s})^{2}+V\left(S^{2}, x\right) d^{D} x \\
+\frac{1}{2} \int \delta \rho\left(S^{2}, x\right) K\left(x, x^{\prime}\right) \delta \rho\left(S^{\prime 2}, x^{\prime}\right) d^{D} x d^{D} x^{\prime}, \\
\int\left[\rho\left(S^{2}, x\right)-\bar{\rho}\right] d^{D} x=0,
\end{gathered}
$$

where the explicit coordinate dependence of the local part of the potential energy $V$ and the charge density increment $\delta \rho\left(S^{2}, x\right) \equiv \rho\left(S^{2}, x\right)-\bar{\rho}$ account for the presence of all other components $u_{i}^{(0)}(x), 2 \leqslant i \leqslant N$ of the order parameter. The expansion (10) remains valid even in the present case, and we can always select the ground-state configuration of the functional $(\mathrm{C} 1)$ to have only one component, $\mathbf{s}=\left(s_{0}(x), 0\right)$. As before, our task is to prove that this configuration is locally unstable to "untwisting," as long as the function $s_{0}(x)$ has a node. The problem with the charge constraint (C2) is slightly more difficult, since the naively perturbed configuration $\mathbf{s}=\left(s_{0}, s_{1}\right)$ generally has a different value of charge. To correct this, we consider a perturbed solution of the form

$$
\mathbf{s}=\left\{s_{0} \sqrt{1-\epsilon_{1}}, \epsilon_{2} w\right\}, \quad S^{2}=s_{0}^{2}+\epsilon_{2}^{2} w^{2}-\epsilon_{1} s_{0}^{2},
$$

where $\epsilon_{1}$ must be chosen to preserve the average charge density, i.e.,

$$
\epsilon_{1}=-\epsilon_{2}^{2}\left[\int \rho^{\prime} w^{2} d^{D} x\right]\left[\int \rho^{\prime} s_{0}^{2} d x\right]^{-1},
$$

where we assume that the denominator does not vanish identically, and the derivative $\rho^{\prime} \equiv \partial \rho\left(S^{2}, x\right) / \partial\left(S^{2}\right)$.

To quadratic order in $\epsilon_{2}$, the increment of the energy functional $(\mathrm{C} 1)$ is just

$$
\begin{aligned}
\delta \mathcal{F}= & \int d x\left\{\chi_{0}(x)\left[\epsilon_{2}^{2}(\nabla w)^{2}-\epsilon_{1}\left(\nabla s_{0}\right)^{2}\right]+\left[\epsilon_{2}^{2} w^{2}-\epsilon_{1} s_{0}^{2}\right]\right. \\
& \left.\times\left[\rho_{0}^{\prime} \varphi_{0}(x)+V_{0}^{\prime}+\left(\nabla s_{0}\right)^{2} \chi_{0}^{\prime}\right]\right\}
\end{aligned}
$$

where all functions with subscript 0 are evaluated with the nonperturbed configurations $s_{0}$, the prime denotes the derivatives over $S^{2}$ as in Eq. (C3), and the scalar potential

$$
\varphi_{0}(x) \equiv \varphi\left(\left[s_{0}^{2}\right], x\right) \equiv \int K\left(x, x^{\prime}\right) \delta \rho\left(s_{0}^{2}\left(x^{\prime}\right), x^{\prime}\right) d^{D} x^{\prime}
$$

Equation (C4) can be simplified with the help of the relation (C3) and the Euler-Lagrange equation for the nonperturbed solution $s_{0}$,

$$
-\nabla\left(\chi_{0} \nabla s_{0}\right)+G\left(\left[s_{0}^{2}\right], x\right) s_{0}=0,
$$

where the self-consistent potential function

$$
G\left(\left[v_{0}^{2}\right], x\right) \equiv\left[\varphi_{0}(x)+\mu\right] \rho_{0}^{\prime}+V_{0}^{\prime}+\left(\nabla s_{0}\right)^{2} \chi_{0}^{\prime}
$$

contains the Lagrange multiplier $\mu$. We obtain, with the same accuracy,

$$
\delta \mathcal{F}=\epsilon_{2}^{2} \int d^{D} x\left\{\chi_{0}(\nabla w)^{2}+G\left(\left[s_{0}^{2}\right], x\right) w^{2}\right\}
$$

Let us return to the Euler-Lagrange equation (C5). As it stands, it is a nonlinear integro-differential equation for $s_{0}$. However, one can formally look at this expression as an action of the linear self-adjoint operator $\hat{L}=-\nabla\left(\chi_{0} \nabla\right)$ $+G_{0}(x) \quad$ (with fixed functions $\chi_{0}(x)$ and $G_{0}(x)$ $\left.\equiv G\left(\left[s_{0}^{2}\right], x\right)\right)$ on the function $s_{0}$. From this point of view $s_{0}$ is an eigenfunction of this operator, $\hat{L} s_{0}=\Lambda_{0} s_{0}$ with zero eigenvalue $\Lambda_{0}=0$. The same operator serves as the kernel of the energy increment (C6), and so, expanding $w=\sum A_{l} s_{l}(x)$ over the orthogonal eigenfunctions of this operator, we obtain 


$$
\delta \mathcal{F}=\epsilon_{2}^{2} \sum \Lambda_{l} A_{l}^{2} \int s_{l}^{2} d^{D} x
$$

By assumption, $s_{0}$ has a node, and so there must ${ }^{72}$ exist an eigenfunction $s_{-1}$ corresponding to a negative eigenvalue $\Lambda_{-1}<0$. Therefore, taking $w=s_{-1}$, we can decrease the free energy,

$$
\Delta \mathcal{F}=\Lambda_{-1} \epsilon_{2}^{2} \int v_{-1}^{2} d^{D} x<0,
$$

which violates the original assumption. Therefore, the spin configurations with nodes are locally unstable to untwisting even in systems with charge constraint and/or long-range interactions.

\section{APPENDIX D: NO-NODE THEOREM FOR DISCRETE SYSTEMS}

It is also possible to prove a version of the no-node theorem for many lattice models. Consider the problem of finding a minimum of the expression

$$
\mathcal{H}=\sum_{i j} \chi_{i j}\left(u_{i}-u_{j}\right)^{2}+V\left(u_{1}^{2}, \ldots, u_{N}^{2}\right),
$$

where the variables $u_{i}, i=1, \ldots, N$ are scalars, ${ }^{92}$ the nonlocal potential $V\left(u_{1}^{2}, \ldots, u_{N}^{2}\right)$ is a limited, continuously differentiable function of all its arguments, and the connections $\chi_{i j} \geqslant 0$ can be positive or zero, with the only limitation that all points can be linked. We are going to prove that in the minimum of Eq. (D1) all variables $u_{i}$ are nonzero and have the same sign, or all of them vanish identically.

Let us suppose that the opposite statement is true, namely, that the global minimum $\mathcal{H}^{(0)}$ is achieved on the set $u_{i}^{(0)}$, some of which could be positive, negative, or zeros, but at least one nonzero value exists. Without limiting generality, we can suppose that this value is positive. Let us now replace the original set by the non-negative set $u_{i}^{(1)}=\left|u_{i}^{(0)}\right|$. Clearly, because of the obvious inequality (Cauchy)

$$
(a-b)^{2} \geqslant(|a|-|b|)^{2},
$$

this substitution cannot increase the energy. This inequality becomes strict if $a$ and $b$ have opposite signs, which implies that the points with positive and negative values in the original configuration must be separated by zeros, or our assumption was wrong. Therefore, some of the values in the modified set $u_{i}^{(1)}$ are expected to be zeros. By assumption, there are no disconnected points, and at least one point $j$ with zero value $u_{j}^{(1)}=u_{j}^{(0)}=0$ must be connected to a point $i$ with $u_{i}^{(1)}>0$. If we replace the zero by a sufficiently small value $u_{j}^{(2)}=\epsilon>0$, the increment of the energy (D1) will be negative,

$$
\begin{aligned}
\delta \mathcal{H}_{j} & =\sum_{i}\left\{\chi_{i j}\left(\epsilon^{2}-2 \epsilon u_{i}^{(1)}\right)\right\}+\left.\epsilon^{2} \frac{\partial V\left(u_{1}^{2}, \ldots\right)}{\partial u_{j}^{2}}\right|_{u_{l}=u_{l}^{(1)}} \\
& =-2 \epsilon \sum_{i} \chi_{i j} u_{i}^{(1)}+\mathcal{O}\left(\epsilon^{2}\right)<0 .
\end{aligned}
$$

The procedure can be repeated for all points with zero value. Therefore, the original assumption was wrong, and in the global minimum all values $u_{i}$ must have the same sign (although they can be exponentially small).

Because the increment (D2) of the energy is linear in $\epsilon$, the proven statement can be easily extended to accommodate an arbitrary dependence of the connections $\chi_{i j}\left(u_{1}^{2}, \ldots, u_{N}^{2}\right)$ on the variables, as well as an arbitrary number of nonlocal constraints of the form $A\left(u_{1}^{2}, \ldots, u_{N}^{2}\right)=0$.
${ }^{1}$ L.P. Pryadko, S. Kivelson, and D.W. Hone, Phys. Rev. Lett. 80, 5651 (1998).

${ }^{2}$ C.S. Hellberg and E. Manousakis, Phys. Rev. Lett. 78, 4609 (1997).

${ }^{3}$ C.S. Hellberg and E. Manousakis, J. Phys. Chem. Solids 59, 1818 (1998).

${ }^{4}$ For a review, see S.A. Kivelson and V.J. Emery, in The Los Alamos Symposium-1993: Strongly Correlated Electronic Materials, edited by K.S. Bedell, Ziqiang Wang, David E. Meltzer, Alexander V. Balatsky, and Elihu Abrahams (Addison-Wesley, New York, 1994), p. 619.

${ }^{5}$ U. Löw, V.J. Emery, K. Fabricius, and S.A. Kivelson, Phys. Rev. Lett. 72, 1918 (1994).

${ }^{6}$ M. Seul and D. Andelman, Science 267, 476 (1995).

${ }^{7}$ J.M. Tranquada, J. Phys. Chem. Solids 59, 2150 (1998).

${ }^{8}$ H. Hwang and S.-W. Cheong, Nature (London) 389, 942 (1997).

${ }^{9}$ S. Mori, C. Chen, and S.-W. Cheong, Nature (London) 392, 473 (1998).

${ }^{10}$ Y.S. Lee, R. J. Birgeneau, M. A. Kastner, Y. Endoh, S. Wakimoto, K. Yamada, R. W. Erwin, S.-H. Lee, and G. Shirane, Phys. Rev. B 60, 3643 (1999).

${ }^{11}$ S. Wakimoto, K. Yamada, S. Ueki, G. Shirane, Y. S. Lee, S. H.
Lee, M. A. Kastner, K. Hirota, P. M. Gehring, Y. Endoh, and R. J. Birgeneau, cond-mat/9902319 (unpublished).

${ }^{12}$ J.M. Tranquada, Physica B 241-243, 745 (1997).

${ }^{13}$ It is possible to have a continuous phase transition between a topologically and a nontopologically stripe-ordered phases. As discussed in Appendix A, such a transition can be interpreted as a dimerization transition, which gives a precise macroscopic meaning to the concept of dimerized stripes.

${ }^{14}$ O. Zachar, S.A. Kivelson, and V.J. Emery, Phys. Rev. B 57, 1422 (1998).

${ }^{15}$ V.J. Emery and S.A. Kivelson, Physica C 209, 597 (1993).

${ }^{16}$ C.S. Hellberg and E. Manousakis, Phys. Rev. Lett. 83, 132 (1999).

${ }^{17}$ P.W. Anderson, Science 235, 1196 (1987).

${ }^{18}$ V J. Emery, Phys. Rev. Lett. 58, 2794 (1987).

${ }^{19}$ P.B. Visscher, Phys. Rev. B 10, 943 (1974).

${ }^{20}$ V.J. Emery, S.A. Kivelson, and H.Q. Lin, Phys. Rev. Lett. 64, 475 (1990)

${ }^{21}$ M. Marder, N. Papanicolaou, and G.C. Psaltakis, Phys. Rev. B 41, 6920 (1990).

${ }^{22}$ S.A. Kivelson and V.J. Emery, Synth. Met. 80, 151 (1996).

${ }^{23}$ C. Nayak and F. Wilczek, Phys. Rev. Lett. 78, 2465 (1997). 
${ }^{24}$ S.R. White and D.J. Scalapino, Phys. Rev. Lett. 81, 3227 (1998).

${ }^{25}$ S.R. White and D.J. Scalapino, Phys. Rev. Lett. 80, 1272 (1998).

${ }^{26}$ S.A. Kivelson, E. Fradkin, and V.J. Emery, Nature (London) 393, 550 (1998).

${ }^{27}$ M. Calandra, F. Becca, and S. Sorella, Phys. Rev. Lett. 81, 5185 (1998)

${ }^{28}$ H. Viertio and T. Rice, J. Phys.: Condens. Matter 6, 7091 (1994).

${ }^{29}$ H.B.G. Casimir, Proc. K. Ned. Akad. Wet. 51, 793 (1948).

${ }^{30}$ J.M. Tranquada, B. J. Sternlieb, J. D. Axe, Y. Nakamura, and S. Uchida, Nature (London) 375, 561 (1995).

${ }^{31}$ M. von Zimmermann, A. Vigliante, T. Frello, J. Madsen, D. J. Buttrey, N. H. Andersen, J. R. Schneider, D. Gibbs, and J. M. Tranquada, J. Supercond. 10, 447 (1997).

${ }^{32}$ A. Vigliante, M. von Zimmermann, J. R. Schneider, T. Frello, N. H. Andersen, J. Madsen, D. J. Buttrey, D. Gibbs, and J. M. Tranquada, Phys. Rev. B 56, 8248 (1997).

${ }^{33}$ M. von Zimmermann, A. Vigliante, T. Niemoller, T. Frello, J. Madsen, P. Wochner, S. Uchida, N. H. Andersen, J. M. Tranquada, D. Gibbs, and J. R. Schneider, Europhys. Lett. 41, 629 (1998).

${ }^{34}$ J.M. Tranquada, J. D. Axe, N. Ichikawa, A. R. Moodenbaugh, Y. Nakamura, and S. Uchida, Phys. Rev. Lett. 78, 338 (1997).

${ }^{35}$ J.E. Ostenson, S. Bud'ko, M. Breitwisch, D. K. Finnemore, N. Ichikawa, and S. Uchida, Phys. Rev. B 56, 2820 (1997).

${ }^{36}$ G.M. Luke, K. Kojima, M. Larkin, J. Merrin, B. Nachumi, Y. J. Uemura, Y. Nakamura, S. Uchida, and M. Crawford, Hyperfine Interact. 105, 113 (1997).

${ }^{37}$ W. Wagener, H.-H. Klauss, M. Hillbe, M. A. C. de Melo, M. Birke, F. J. Littrest, B. Buchner, and H. Micklitz, Phys. Rev. B 55, R14 761 (1997).

${ }^{38}$ B. Nachumi, Y. Fudamoto, A. Keren, K. M. Kojima, M. Larkin, G. M. Luke, J. Merrin, O. Tchernyshyov, Y. J. Uemura, N. Ichikawa, M. Goto, H. Takagi, S. Uchida, M. K. Crawford, E. M. McCarron, D. E. MacLaughlin, and R. H. Heffner, Phys. Rev. B 58, 8760 (1998).

${ }^{39}$ B. Büchner, M. Cramm, M. Braden, W. Bravnisch, O. Hoffels, W. Schnelle, R. Muller, A. Freimuth, W. Schlabitz, G. Heger, D. I. Khomskii, and D. Wohlleben, Europhys. Lett. 21, 953 (1993).

${ }^{40}$ B. Büchner, M. Breuer, A. Freimuth, and A.P. Kampf, Phys. Rev. Lett. 73, 1841 (1994).

${ }^{41}$ S.-W. Cheong, G. Aeppli, T. E. Mason, H. Mook, S. M. Hayden, P. C. Canfield, Z. Fisk, K. N. Clausen, and J. L. Martinez, Phys. Rev. Lett. 67, 1791 (1991).

${ }^{42}$ H.A. Mook, F. Dogan, and B.C. Chakoumakos, cond-mat/9811100 (unpublished).

${ }^{43}$ Z.-X. Shen, P. J. White, D. L. Feng, C. Kim, G. D. Gu, H. Ikeda, R. Yoshizaki, and N. Koshizuka, Science 280, 259 (1998).

${ }^{44}$ C. Niedermayer, T. Bernhard, T. Blasius, A. Golnik, A. Moodenbaugh, and J. I. Budnick, Phys. Rev. Lett. 80, 3843 (1998).

${ }^{45}$ A.W. Hunt, P.M. Singer, K.R. Thurber, and T. Imai, Phys. Rev. Lett. 82, 4300 (1999).

${ }^{46}$ C.H. Chen, S.-W. Cheong, and A.S. Cooper, Phys. Rev. Lett. 71, 2461 (1993).

${ }^{47}$ J.M. Tranquada, J.E. Lorenzo, D.J. Buttrey, and V. Sachan, Phys. Rev. B 52, 3581 (1995).

${ }^{48}$ J.M. Tranquada, D.J. Buttrey, and V. Sachan, Phys. Rev. B 54, 12318 (1996).

${ }^{49}$ S.-H. Lee and S.-W. Cheong, Phys. Rev. Lett. 79, 2514 (1997).

${ }^{50}$ J. Zaanen and O. Gunnarsson, Phys. Rev. B 40, 7391 (1989).

${ }^{51}$ J. Zaanen and P.B. Littlewood, Phys. Rev. B 50, 7222 (1994).

${ }^{52}$ H. Schulz, J. Phys. (Paris) 50, 2833 (1989).
${ }^{53}$ A.J. Heeger, S.A. Kivelson, J.R. Schrieffer, and W.-P. Su, Rev. Mod. Phys. 60, 781 (1988).

${ }^{54}$ J.P. Lu, Q. Si, J.H. Kim, and K. Levin, Physica C 179, 191 (1991).

${ }^{55}$ K. Yamada, C. H. Lee, K. Kurahashi, J. Wada, S. Wakimoto, S. Ueki, H. Kimura, Y. Endoh, S. Hosoya, G. Shirane, R. J. Birgeneau, M. Greven, M. A. Kastner, and Y. J. Kim, Phys. Rev. B 57, 6165 (1998). The data here do not really look linear for small $x$, but show some curvature, which indicates a slight variation of the stripes' filling with the doping.

${ }^{56}$ A.V. Fedorov, T. Valla, P. D. Johnson, Q. Li, G. D. Gu, and N. Koshizuka, Phys. Rev. Lett. 82, 2179 (1999).

${ }^{57}$ J. Schrieffer, X.-G. Wen, and S.-C. Zhang, Phys. Rev. Lett. 60, 944 (1988).

${ }^{58}$ S.-C. Zhang, Science 275, 1089 (1997).

${ }^{59}$ M. Veillette, Y.B. Bazaliy, A.J. Berlinsky, and C. Kallin, cond-mat/9812282 (unpublished).

${ }^{60}$ R. Dashen, B. Hasslacher, and A. Neveu, Phys. Rev. D 10, 4114 (1974).

${ }^{61}$ N. Christ and T. Lee, Phys. Rev. D 12, 1606 (1975).

${ }^{62}$ R. Friedberg, T. Lee, and A. Sirlin, Nucl. Phys. B 115, 1 (1976).

${ }^{63}$ R. Rajaraman and E. Weinberg, Phys. Rev. D 11, 2950 (1975).

${ }^{64}$ R. Friedberg, T. Lee, and A. Sirlin, Phys. Rev. D 13, 2739 (1976).

${ }^{65}$ V.J. Emery, A.H. Luther, and I. Peschel, Phys. Rev. B 13, 1272 (1976).

${ }^{66}$ R. Rajaraman, Phys. Rev. Lett. 42, 200 (1979).

${ }^{67}$ H. Schiff, Phys. Rev. D 25, 1048 (1982).

${ }^{68} \mathrm{R}$. Rajaraman, Solitons and Instantons: An Introduction to Solitons and Instantons in Quantum Field Theory (North-Holland, New York, 1982).

${ }^{69}$ Although the long-distance interaction favors uniformly charged configurations, one can show that periodic configurations necessarily replace the phase separation as long as this interaction is sufficiently weak.

${ }^{70}$ L.D. Landau and E.M. Lifshitz, Quantum Mechanics: Nonrelativistic Theory (Pergamon Press, Oxford, 1977).

${ }^{71} \mathrm{~A}$ generalization to systems with charge constraint is considered in Appendix B; also see related analysis in Sec. V.

${ }^{72}$ R. Courant and D. Hilbert, in Methods of Mathematical Physics (Interscience, New York, 1953), Vol. I, Chap. VI.

${ }^{73}$ Usually, when analyzing the stability of a system with a singlecomponent order parameter, the translational symmetry is used to find the zero-energy solution of Eq. (12). The corresponding solution is the derivative of the original function $s_{0}(x)$, and the local stability implies that the function $s_{0}^{\prime}(x)$ has no nodes, which is impossible for any nontrivial solution under periodic boundary conditions (but it does happen for topologically stable solutions). In our case the one-component translational symmetry is broken by the coupling to the remaining nonuniform components $\phi_{i}(x)$ of the order parameter, as indicated by the explicit coordinate dependence of the parameters in Eq. (11).

${ }^{74}$ It was substantial that all physical quantities are continuously differentiable functions of $s^{2}$, while most generally the timereversal symmetry only requires the dependence on the magnitude $|s|$. Such a requirement is never a problem if $s$ is a component of a magnetic order parameter. In general, continuity implies that the proven statement can be violated only if the lowest eigenvalue of Eq. (12) diverges in a singular limit, $\Lambda_{-1}$ $\rightarrow-\infty$.

${ }^{75}$ Invariants quadratic in $S_{i}$ and linear in derivatives also exist for 
seven point groups $C_{6 v}, C_{4 v}, C_{3 v}, C_{2 v}, D_{2 d}, S_{4}$, and $C_{s}$ which do not contain inversion. Three remaining groups without inversion, $T_{d}, D_{3 h}$, and $C_{3 h}$, admit only invariants quartic in $S_{i}$ and linear in derivatives.

${ }^{76}$ D.E. Cox, A.R. Moodenbaugh, J.J. Hurst, and R.H. Jones, in Novel Superconductivity. Proceedings of the International Workshop on Novel Mechanisms of Superconductivity, edited by S.A. Wolf and V.Z. Kresin (Plenum, New York, 1987), pp. 746-747.

${ }^{77}$ D.E. Cox, A.R. Moodenbaugh, J.J. Hurst, and R.H. Jones, J. Phys. Chem. Solids 49, 47 (1988).

${ }^{78}$ J.D. Axe, A. H. Moudden, D. Hohlwein, D. E. Cox, K. M. Mohanty, A. R. Moodenbaugh, and Xu Youwen, Phys. Rev. Lett. 62, 2751 (1989).

${ }^{79}$ J.D. Axe, D. E. Cox, K. Mohanty, H. Moudden, A. R. Moodenbaugh, Xu Youwen, and T. R. Thurston, IBM J. Res. Dev. 33, 382 (1989).

${ }^{80}$ D.E. Cox, P. Zolliger, J. D. Axe, A. H. Moudden, A. R. Moodenbaugh, and Y. Xu, in High Temperature Superconductors: Relationships Between Properties, Structure, and Solid-State Chemistry, edited by J.D. Jorgensen, K. Kitezawa, J.M. Taras- con, M.S. Thompson, and J.B. Torrance, MRS Symposia Proceedings No. 156 (Materials Research Society, Pittsburgh, 1989), pp. 141-151.

${ }^{81}$ Y. Zhu, A. R. Moodenbaugh, Z. X. Cai, J. Tafto, M. Suenaga, and D. O. Welch, Phys. Rev. Lett. 73, 3026 (1994).

${ }^{82}$ A.R. Moodenbaugh, Wu Lijun, Zhu Yimei, L. H. Lewis, and D. E. Cox, Phys. Rev. B 58, 9549 (1998).

${ }^{83}$ A. H. Castro Neto, Z. Phys. B 103, 185 (1997).

${ }^{84}$ A.H. Castro Neto and D. Hone, Phys. Rev. Lett. 76, 2165 (1996).

${ }^{85}$ J. Zaanen, J. Phys. Chem. Solids 59, 1769 (1998).

${ }^{86}$ S. Wakimoto, G. Shirane, Y. Endoh, K. Hirota, S. Ueki, K. Yamada, R. J. Birgeneau, M. A. Kastner, Y. S. Lee, P. M. Gehring, and S. H. Lee, Phys. Rev. B 60, R769 (1999).

${ }^{87} \mathrm{~S}$. Wakimoto (private communication).

${ }^{88}$ Y.B. Bazaliy and L.P. Pryadko (unpublished).

${ }^{89}$ N. Manton, Nucl. Phys. B 126, 525 (1977).

${ }^{90}$ J. Jersak, M. Kiera, and M. Magg, Nuovo Cimento A 40, 269 (1977).

${ }^{91}$ A. Abanov and V.L. Pokrovsky, Phys. Rev. B 58, R8889 (1998).

${ }^{92}$ The generalization to a multicomponent order parameter is straightforward. 